\title{
Environmental balance of the UK biogas sector: An evaluation by consequential life cycle assessment
}

Styles, David; Dominguez, Eduardo Mesa; Chadwick, Dave

\section{Science of the Total Environment}

DOI:

10.1016/j.scitotenv.2016.03.236

Published: 01/08/2016

Peer reviewed version

Cyswllt i'r cyhoeddiad / Link to publication

Dyfyniad o'r fersiwn a gyhoeddwyd / Citation for published version (APA):

Styles, D., Dominguez, E. M., \& Chadwick, D. (2016). Environmental balance of the UK biogas sector: An evaluation by consequential life cycle assessment. Science of the Total Environment, 560-561, 241-253. https://doi.org/10.1016/j.scitotenv.2016.03.236

\footnotetext{
Hawliau Cyffredinol / General rights

Copyright and moral rights for the publications made accessible in the public portal are retained by the authors and/or other copyright owners and it is a condition of accessing publications that users recognise and abide by the legal requirements associated with these rights.

- Users may download and print one copy of any publication from the public portal for the purpose of private study or research.

- You may not further distribute the material or use it for any profit-making activity or commercial gain

- You may freely distribute the URL identifying the publication in the public portal?
}

Take down policy

If you believe that this document breaches copyright please contact us providing details, and we will remove access to the work immediately and investigate your claim. 


\section{Environmental balance of the UK biogas sector: an}

\section{3 evaluation by consequential life cycle assessment}

4 David Styles †*, Eduardo Mesa Dominguez $†$, Dave Chadwick†

$5 \quad *$ Corresponding author: d.styles@bangor.ac.uk +44(0)1248 382502.

$6 \uparrow$ School of Environment, Natural Resources and Geography, Bangor, Gwynedd, UK, LL57

$72 \mathrm{UW}$.

8 KEYWORDS: anaerobic digestion, GHG, LCA, waste management, bioenergy, resource

9 efficiency 
17 Anaerobic digestion (AD) is expanding rapidly in the UK. Previous life cycle assessment (LCA)

18 studies have highlighted the sensitivity of environmental outcomes to feedstock type, fugitive

19 emissions, biomethane use, energy conversion efficiency and digestate management. We

20 combined statistics on current and planned AD deployment with operational data from a survey

21 of biogas plant operators to evaluate the environmental balance of the UK biogas sector for the

22 years 2014 and 2017. Consequential LCA was applied to account for all major environmental

23 credits and burdens incurred, including: (i) substitution of composting, incineration, sewer

24 disposal, field decomposition and animal feeding of wastes; (ii) indirect land use change (ILUC)

25 incurred by the cultivation of crops used for biogas production and to compensate for bakery and

26 brewery wastes diverted from animal feed. In 2014, the UK biogas sector reduced greenhouse

27 gas (GHG) emissions by 551-755 Gg CO $2 \mathrm{e}$ excluding ILUC, or 238-755 $\mathrm{Gg} \mathrm{CO}_{2} \mathrm{e}$ including

28 ILUC uncertainty. Fossil energy depletion was reduced by 8.9-10.8 PJe, but eutrophication and

29 acidification burdens were increased by 1.8-3.4 $\mathrm{Gg} \mathrm{PO}_{4} \mathrm{e}$ and 8.1-14.6 $\mathrm{Gg} \mathrm{SO}_{2} \mathrm{e}$, respectively.

30 Food waste and manure feedstocks dominate GHG abatement, largely through substitution of in-

31 vessel composting and manure storage, whilst food waste and crop feedstocks dominate fossil

32 energy credit, primarily through substitution of natural gas power generation. Biogas expansion

33 is projected to increase environmental credits and loadings by a factor of 2.4 by 2017 . If all AD

34 bioelectricity replaced coal generation, or if $90 \%$ of biomethane replaced transport diesel or grid

35 natural gas, GHG abatement would increase by 131\%, 38\% and 20\%, respectively. Policies to

36 encourage digestion of food waste and manures could maximize GHG abatement, avoiding the

37 risk of carbon leakage associated with use of crops and wastes otherwise used to feed livestock.

38 Covering digestate stores could largely mitigate net eutrophication and acidification burdens. 
Anaerobic digestion (AD) is an established technology to treat wet organic wastes that is

41 increasingly being deployed as a renewable energy technology across Europe to convert a range

42 of feedstocks into biogas and ultimately bioelectricity, bioheat or transport fuel. This has arisen

43 largely in response to energy-related subsidies paid to AD operators, such as feed-in-tariffs

44 (FITs) paid for bioelectricity and the Renewable Heat Incentive (RHI) paid for bioheat (OFGEM,

45 2016). Up until 2014, almost all biogas produced in the UK was used to fuel combined heat and

46 power (CHP) engines, producing bioelectricity eligible for a FIT subsidy of up to $£ 0.10 / \mathrm{kWh}$ in

472015 (OFGEM, 2015). In 2014, there were 32 small-scale plants ( $\leq 250 \mathrm{kWe})$ with a cumulative

48 installed capacity of $3.4 \mathrm{MWe}, 48$ medium scale plants $(>250 \mathrm{kWe} \leq 500 \mathrm{kWe})$ with a cumulative

49 installed capacity of $22.6 \mathrm{MWe}$, and 72 large-scale plants $(>500 \mathrm{kWe})$ with a cumulative installed

50 capacity of $136.0 \mathrm{MWe}(\mathrm{NNFCC}, 2014)$. The overall efficiency of electricity generation can vary

51 significantly across AD-CHP plants (Bacenetti et al., 2013), influencing their performance in

52 terms of greenhouse gas (GHG) abatement and fossil fuel substitution. Similarly, the magnitude

53 of eutrophication and acidification burdens arising largely from ammonia $\left(\mathrm{NH}_{3}\right)$ emissions

54 during the storage and field application of digestate are highly dependent on the type digestate

55 storage infrastructure and method of digestate application (Boulamanti et al., 2013: Styles et al.,

$562015 \mathrm{a} ; \mathrm{b})$. However, up until now there has been little or no published information on the

57 conversion efficiency of biogas feedstock into electricity, or on the methods of storing and

58 spreading the residual digestate produced, for the UK biogas sector as a whole.

59 A review of 15 attributional life cycle assessment (LCA) studies (Hijazi et al., 2016) found that

60 biogas energy has a lower GHG intensity than fossil reference energy, but can increase

61 acidification and eutrophication burdens. Whiting and Azapagig (2014) reported that biogas 
62 produced from agricultural wastes resulted in significant GHG abatement when it substituted

63 natural gas in CHP engines, but increased acidification and eutrophication burdens by 25 and 12

64 fold, respectively. Whilst high biogas yields from maize have been found to support generation

65 of bioelectricity with a considerably lower GHG intensity than grid-electricity in Germany and

66 the UK (Whiting and Azapagig, 2014; Claus et al., 2014), maize biogas was also found to

67 increase environmental burdens across eight out of 11 impact categories compared with natural

68 gas (Whiting and Azapagig, 2014). However, fugitive emissions of methane $\left(\mathrm{CH}_{4}\right)$ are highly

69 variable (Adams et al., 2015), and may significantly reduce net GHG abatement achieved by

70 crop-biogas. Furthermore, the aforementioned attributional LCA studies did not expand system

71 boundaries to account for important indirect effects of AD system deployment, including the

72 diversion of organic waste from alternative fates such as landfill or composting, and indirect land

73 use change (ILUC) associated with the displacement of food crop production. Such effects could

74 have an important influence on the environmental outcome of $\mathrm{AD}$ deployment at the national

75 level, and may be captured by consequential LCA that accounts for indirect effects caused by

76 market signals arising from an intervention (Weidema, 2001).

77 Styles et al. (2015a) applied consequential LCA to highlight the risk of carbon leakage from

78 ILUC when animal feed is diverted to biogas plants on dairy farms, building on the recognised

79 need to expand LCA boundaries in order to accurately evaluate the environmental efficiency of

80 biogas and other renewable energy options (Rehl et al., 2012; Tufvesson et al., 2013; Tonini et

81 al., 2016). Numerous recent studies have indicated that crop-biogas may actually increase GHG

82 emissions when replacing fossil energy, if ILUC is incurred (Tonini et al., 2012; Hamelin et al.,

83 2012; Styles et al., 2015b). LCA system boundaries have also been expanded to quantify

84 significant GHG credits for biogas plants attributable to the avoidance of manure storage and 
85 waste management (e.g. composting), and fertilizer replacement by digestate (Borjesson and

86 Berglund, 2007; Bacenetti et al., 2013; Boulamanti et al., 2013). For example, despite limited

87 bioenergy yields (Lijó et al., 2014a), the digestion of pig slurry can achieve GHG abatement of

$88 \quad 1.19 \mathrm{~kg} \mathrm{CO} 2 \mathrm{e}$ per $\mathrm{kWh}$ of electricity generated when avoided slurry storage emissions are

89 accounted for as a credit (Bancenetti et al., 2013).

90 Bywater (2011) characterized small-scale farm AD-CHP plants $\leq 250 \mathrm{kWe}$ capacity in the UK,

91 typically co-digesting animal manures and crops, with single-stage digestion and relatively short

92 residence times, often storing digestate in pre-existing manure storage facilities. Larger AD

93 plants usually digest waste and crop feedstocks, and are more likely to be optimized for

94 electricity generation with e.g. two-stage digestion and longer residence times. Given recent

95 findings on large differences in life cycle environmental performance for different types of

96 feedstock and operating parameters, quantifying the overall environmental balance of $\mathrm{AD}$

97 deployment in the UK could provide useful insight for policy makers on the efficacy of policies

98 relating to $\mathrm{AD}$, in terms of climate change, energy security, and air and water pollution.

99 Consequential LCA is the most relevant approach for such an evaluation because it accounts for

100 direct and indirect environmental burdens and credits associated with the introduction of AD,

101 considering process substitution (Weidema, 2001; Tufvesson et al., 2013; Tonini et al., 2016).

102 A detailed statistical overview of the UK biogas sector has recently been provided by NNFCC

103 (2014a;b), including information on regional deployment, plant scale, feedstock requirements

104 and land requirements, alongside projected biogas capacity development up until 2017.

105 However, that overview did not include information on design and operational parameters

106 critical to environmental performance. We compiled a uniquely detailed profile of the non-

107 wastewater treatment plant (WWTP) biogas sector in the UK by combining plant size and 
108 feedstock data (NNFCC, 2014a;b) with operational data obtained from a survey of UK biogas

109 plants. We then applied consequential LCA to evaluate the net environmental balance of the

110 sector, and to explore alternative scenarios of deployment. This paper presents results from the

111 biogas plant survey, and describes how they were used to estimate the environmental profile of

112 the UK biogas sector through consequential LCA.

\section{1. MATERIALS AND METHODS}

$1142.1 \mathrm{Goal}$, scope and boundary definition

115 The aim of this study was to quantify the net environmental balance (burden $\underline{\text { change) }}$ )

116 associated with recent and projected deployment of $\mathrm{AD}$ in the UK, considering incurred and

117 avoided processes and emissions. The study involved the development and application of the

$118 L C A D$ EcoScreen tool, applying a consequential LCA approach to calculate the life cycle

119 environmental balance of $\mathrm{AD}$ in relation to a reference flow of one $\mathrm{Mg}$ of dry matter (DM)

120 feedstock input, for a range of characterized feedstocks, plant operating characteristics and

121 counterfactual (substituted) material flows. Major processes accounted for are shown in Figure 1.

122 The construction and manufacture of buildings and equipment were excluded from the scope, as

123 is typical for bioenergy carbon footprints (EC, 2009). 


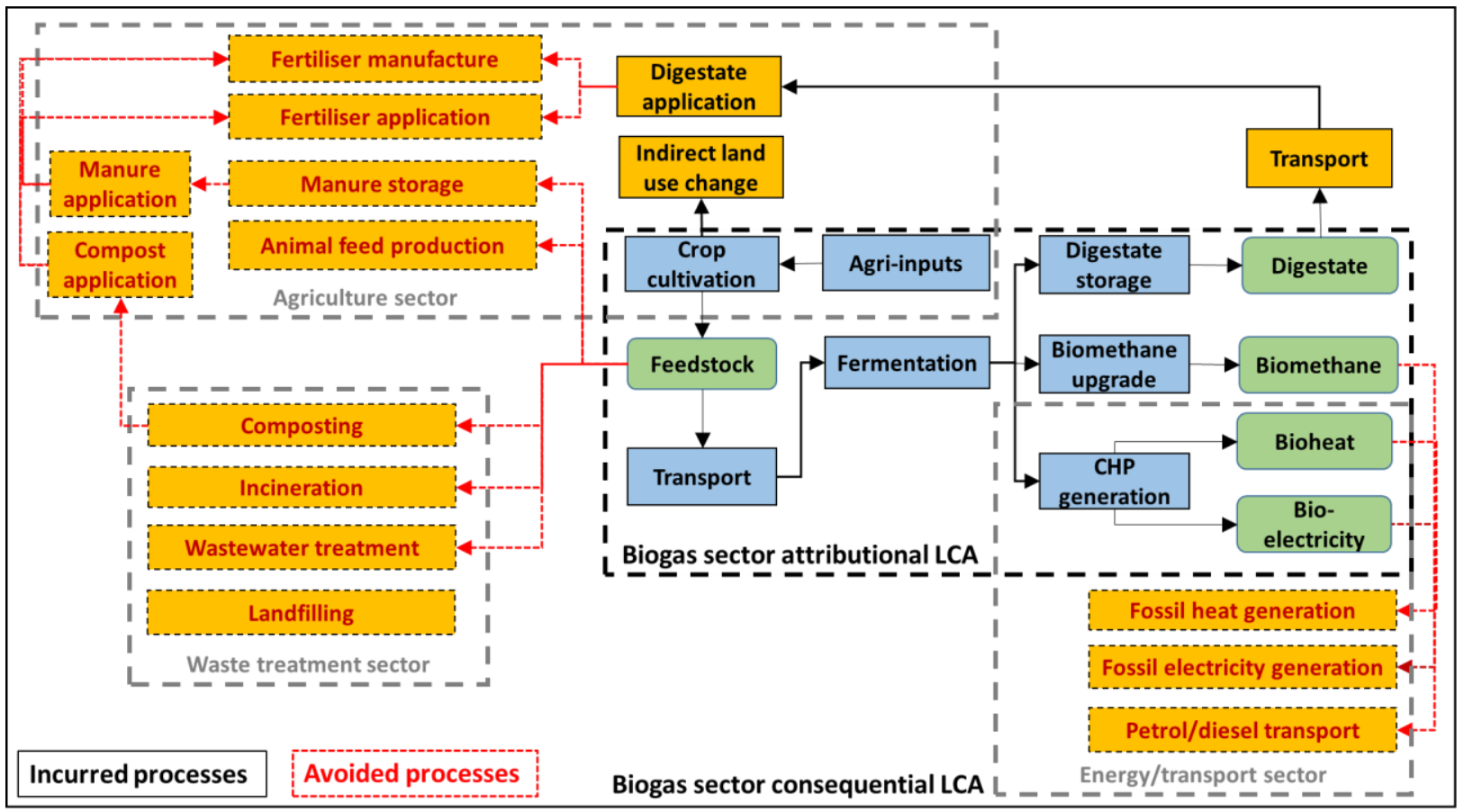

125 Figure 1. Simplified schematic representation of the major processes considered within the 126 consequential LCA undertaken in this study, compared with an attributional LCA boundary.

128 Results were expressed in relation to four environmental impact categories based on CML

129 (2010) characterization factors: global warming potential (GWP) expressed as $\mathrm{CO}_{2} \mathrm{e}$,

130 eutrophication potential (EP) expressed as $\mathrm{PO}_{4} \mathrm{e}$, acidification potential (AP) expressed as $\mathrm{SO}_{2} \mathrm{e}$,

131 and fossil resource depletion potential (FRDP) expressed as MJe. For example, 100-yr GWP

132 factors for $\mathrm{CH}_{4}$ and $\mathrm{N}_{2} \mathrm{O}$ are 25 and 298, respectively. Scenario results were normalised against

133 reported annual UK loadings for GWP (DECC, 2015), and against estimated UK loadings for

134 EP, AP and FRDP after per capita extrapolation of EU loadings (Sleeswijk et al., 2008) based on

135 EU 28 and UK population numbers of 510 million and 65 million, respectively (Eurostat, 2016). 
138 LCA was undertaken for $1 \mathrm{Mg}$ DM across 16 types of feedstock, and a total of 77 permutations

139 of feedstock and plant operating parameters, based on national quantities of biogas feedstocks

140 and plant operating characteristics described in the subsequent sections. Table 1 summarises the

141 framework methodology employed, modified from Styles et al. (2015a;b). Inputs accounted for

142 in detailed attributional LCA studies found to be of minor relevance to the four impact categories

143 studied, such as water, lubricating oil and sodium hydroxide (e.g. Poeschl et al., 2012), were

144 disregarded; the aim was to accurately capture wider environmental effects of the sector as a

145 whole.

147 Table1. Methods applied within the $L C A D$ EcoScreen tool to calculate activity data, emissions 148 and environmental burdens in relation to a reference flow of one $\mathrm{Mg}$ feedstock dry matter

\begin{tabular}{|c|c|c|}
\hline & Process & $\begin{array}{l}\text { Method and data to calculate primary emissions and burdens in relation to } \\
\text { feedstock inputs }\end{array}$ \\
\hline \multirow{7}{*}{ 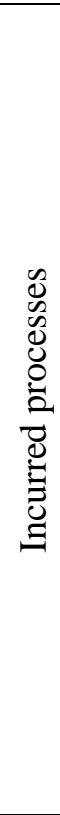 } & Cultivation & $\begin{array}{l}\text { Burdens }=\mathrm{Mg} \text { DM } \times \text { crop cultivation burdens assuming mineral fertiliser application } \\
\text { (Table } 2) \times 1.11 \text { (10\% silage loss }) \text {. }\end{array}$ \\
\hline & $\begin{array}{l}\text { Indirect land } \\
\text { use change }\end{array}$ & $\begin{array}{l}\text { GWP and EP burdens }=\mathrm{Mg} \text { DM / yield, Mg/ha } \mathrm{x} \text { terrestrial } \mathrm{C} \text { and } \mathrm{N} \text { loss per ha at } \\
\text { global agricultural frontier (Styles et al., 2015b), based on IPCC (2006) Tier } 1 \text { EFs. }\end{array}$ \\
\hline & Transport & $\begin{array}{l}\text { Burdens }=\mathrm{Mg} \text { DM } / \mathrm{DM} \% \text { of wet weight (Table } 3) \times 5 \mathrm{~km} / 50 \mathrm{~km} \text { for crops } / \text { wastes } \mathrm{x} \\
\text { Ecoinvent v3.1 burdens per tkm for tractor-trailer } / 16-32 \text { tonne truck (Table } 2 \text { ). }\end{array}$ \\
\hline & $\begin{array}{l}\text { Digester } \\
\text { leakage }\end{array}$ & $\begin{array}{l}\mathrm{kg} \mathrm{CH} \mathrm{CH}_{4}=\mathrm{Mg} \mathrm{DM} \times \mathrm{m}^{3} / \mathrm{Mg} \mathrm{CH}_{4} \text { yield (Table } 3 \text { ) } \times 0.67 \mathrm{~kg} / \mathrm{m}^{3} \times 1 \% \text { digester loss } \\
\text { (Adams et al., 2015). }\end{array}$ \\
\hline & $\begin{array}{l}\text { CHP } \\
\text { combustion }\end{array}$ & $\begin{array}{l}\left.\mathrm{kg} \mathrm{CH}=\mathrm{Mg} \mathrm{DM} \times \mathrm{m}^{3} / \mathrm{Mg} \mathrm{CH} \mathrm{CH}_{4} \text { yield (Table } 3\right) \times 0.67 \mathrm{~kg} / \mathrm{m}^{3}-1 \% \text { digester loss } \mathrm{x} \\
0.5 \% \text { CHP slip. } \\
\mathrm{AP} \text { and EP burdens }=\mathrm{MJ} \mathrm{CH}_{4} \times \text { natural gas CHP burdens from Ecoinvent v3.1 } \\
\text { (Table 2). }\end{array}$ \\
\hline & $\begin{array}{l}\text { Digestate } \\
\text { storage }\end{array}$ & $\begin{array}{l}\left.\mathrm{kg} \mathrm{CH}_{4}=\mathrm{Mg} \mathrm{DM} \mathrm{x} \mathrm{m} / 3 \mathrm{Mg} \mathrm{CH}_{4} \text { yield (Table } 3\right) \times 0.67 \mathrm{~kg} / \mathrm{m}^{3} \times 1.5 \% \text { for medium and } \\
\text { large plants; } \mathrm{CH}_{4} \text { yield } \times 1.5 \% / 4 \% / 9 \% \text { (closed/open tank/lagoon) for small plants. } \\
\mathrm{kg} \mathrm{NH}-\mathrm{N}=\mathrm{Mg} \mathrm{DM} \times \text { total } \mathrm{N}, \mathrm{kg} / \mathrm{Mg} \times \% \text { total } \mathrm{N} \text { as } \mathrm{NH}_{4}-\mathrm{N} \text { (Table 3) } \mathrm{x} \\
2 \% / 10 \% / 52 \% \text { for closed tank/open tank/lagoon (Misselbrook et al., 2012). } \\
\text { Indirect } \mathrm{N}_{2} \mathrm{O}-\mathrm{N}=\mathrm{NH}_{3}-\mathrm{N} \times 0.01 \text { (IPCC, 2006). }\end{array}$ \\
\hline & Digestate & Burdens $=\mathrm{Mg}$ DM / DM \% of wet weight (Table 3$) \times 5 \mathrm{~km}(0$ for manure digestate - \\
\hline
\end{tabular}




\begin{tabular}{|c|c|c|}
\hline & \multirow{2}{*}{$\begin{array}{l}\text { transport } \\
\text { Digestate } \\
\text { application }\end{array}$} & \multirow[b]{2}{*}{ 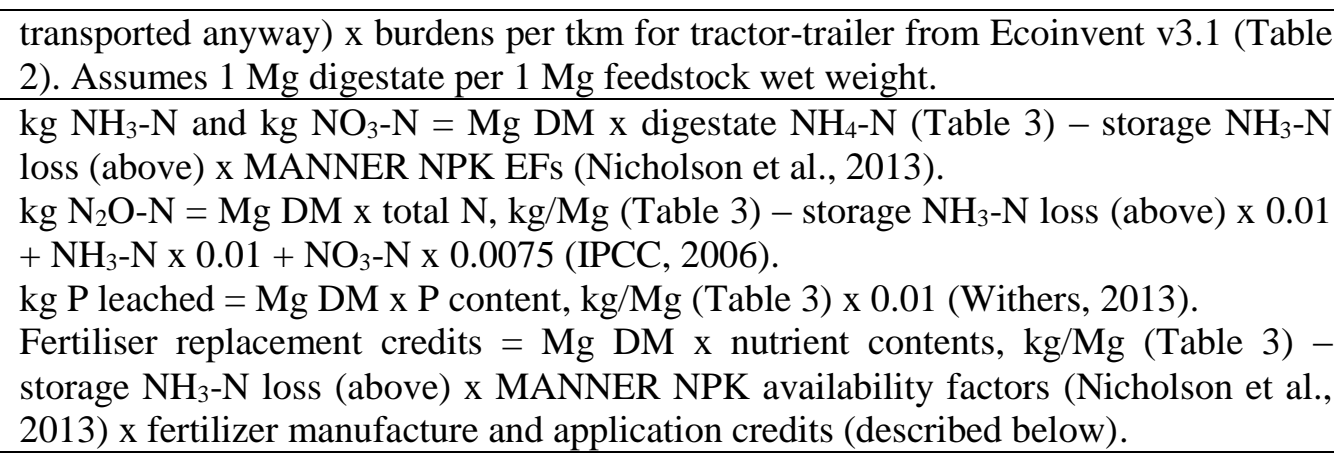 } \\
\hline & & \\
\hline & $\begin{array}{l}\text { Avoided } \\
\text { manure } \\
\text { storage }\end{array}$ & $\begin{array}{l}\text { Avoided } \mathrm{kg} \mathrm{CH} \mathrm{CH}_{4}=\mathrm{Mg} \mathrm{DM} \times 800 \mathrm{~kg} / \mathrm{Mg} \text { volatile solids } \times \mathrm{CH}_{4} \text {-producing capacity for } \\
\text { manure type (IPCC, 2006) x } 0.67 \mathrm{~kg} / \mathrm{m}^{3} \mathrm{CH}_{4} \times \mathrm{CH}_{4} \text { conversion factor by system type } \\
\text { (IPCC, 2006). } \\
\text { Avoided } \mathrm{kg} \mathrm{N} \mathrm{N}_{2} \mathrm{O}-\mathrm{N}=\mathrm{Mg} \mathrm{DM} \times \text { total N, } \mathrm{kg} / \mathrm{Mg} \text { (Table 3) x storage system EFs } \\
\text { (IPCC, 2006). } \\
\text { Avoided } \mathrm{kg} \mathrm{NH}-\mathrm{N}=\mathrm{Mg} \mathrm{DM} \times \text { total N, } \mathrm{kg} / \mathrm{Mg} \text { (Table } 3) \times \% \text { total } \mathrm{N} \text { as } \mathrm{NH}_{4}-\mathrm{N} \\
\text { (Webb and Misselbrook, 2004) x storage system EFs (Misselbrook et al., 2012). } \\
\text { Elaborated in SI 3.1. }\end{array}$ \\
\hline & $\begin{array}{l}\text { Avoided } \\
\text { manure } \\
\text { application }\end{array}$ & 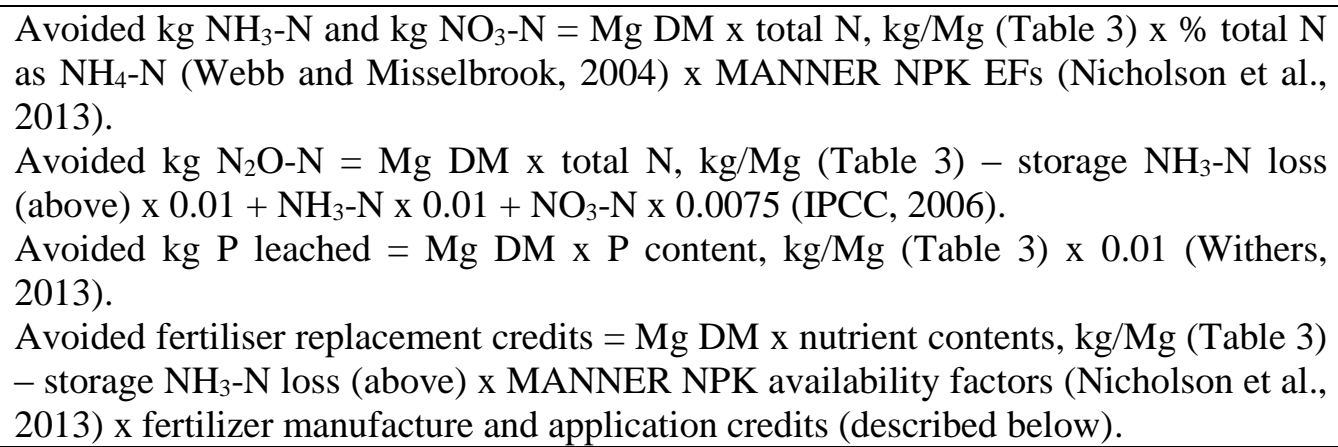 \\
\hline 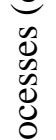 & $\begin{array}{l}\text { Avoided in- } \\
\text { vessel } \\
\text { composting }\end{array}$ & $\begin{array}{l}\text { Avoided emissions, grid electricity use, fertiliser replacement and soil C } \\
\text { sequestration as described in Styles et al. (2015b). Elaborated in SI 3.2. Net credit } \\
\text { values in Table } 2 \text {. }\end{array}$ \\
\hline & $\begin{array}{l}\text { Avoided } \\
\text { landfilling }\end{array}$ & $\begin{array}{l}\text { Avoided emissions, methane capture and grid electricity replacement credits as } \\
\text { described in Styles et al. (2015a). Elaborated in SI 3.3. Net credit values in Table } 2 .\end{array}$ \\
\hline 遂 & $\begin{array}{l}\text { Displaced } \\
\text { animal feed }\end{array}$ & $\begin{array}{l}\text { Additional incurred burdens }=\mathrm{Mg} \text { feedstock DM x Ecoinvent v3.1 burdens for } 1 \mathrm{Mg} \\
\text { wheat-based concentrate feed (Table } 2 \text { ). ILUC effect from compensatory production } \\
\text { calculated as described above for crop feedstock. Elaborated in SI 3.4. }\end{array}$ \\
\hline & $\begin{array}{l}\text { Avoided } \\
\text { field residue } \\
\text { decompositi } \\
\text { on }\end{array}$ & $\begin{array}{l}\text { Avoided emissions, soil } \mathrm{C} \text { sequestration and fertiliser replacement credits described } \\
\text { in SI 3.5. Net credit values in Table } 2 .\end{array}$ \\
\hline & $\begin{array}{l}\text { Avoided } \\
\text { incineration } \\
\text { with energy } \\
\text { recovery }\end{array}$ & $\begin{array}{l}\text { Avoided burdens }=1 \mathrm{Mg} \text { DM animal processing waste } \mathrm{x} \text { Ecoinvent v3.1 burdens for } \\
\text { incineration, corrected for moisture content }- \text { credits for avoided natural gas } \\
\text { electricity generation, elaborated in SI 3.6. Net credit values in Table } 2 \text {. }\end{array}$ \\
\hline & $\begin{array}{l}\text { Avoided } \\
\text { sewer } \\
\text { disposal }\end{array}$ & $\begin{array}{l}\text { Avoided burdens of wastewater treatment including } \mathrm{AD} \text { approximated to } \\
\text { environmental burdens of on-site } \mathrm{AD} \text { - elaborated in SI 3.7. Net credit values in } \\
\text { Table } 2 \text {. }\end{array}$ \\
\hline & $\begin{array}{l}\text { Avoided } \\
\text { marginal } \\
\text { grid }\end{array}$ & $\begin{array}{l}\left.\text { Avoided burdens }=\mathrm{Mg} \mathrm{DM} \mathrm{x} \mathrm{m} 3 / \mathrm{Mg} \mathrm{CH}_{4} \text { yield (Table } 3\right) \times 0.67 \mathrm{~kg} / \mathrm{m}^{3}-1 \% \text { digester } \\
\text { loss }-0.5 \% \text { CHP slip x } 50 \mathrm{MJ} / \mathrm{kg} \mathrm{LHV} \mathrm{x} \mathrm{CHP} \mathrm{elec.} \mathrm{efficiency}(35 \% \text { or } 40 \% \text { for small } \\
\& \text { medium or large scale } \mathrm{AD})-6 \% \text { parasitic load } \mathrm{x} \text { natural gas combined cycle }\end{array}$ \\
\hline
\end{tabular}




\begin{tabular}{|c|c|}
\hline $\begin{array}{l}\text { electricity } \\
\text { generation }\end{array}$ & $\begin{array}{l}\text { electricity generation burdens per MJ generated from Ecoinvent v3.1. Net credit } \\
\text { values in Table } 2 \text {. }\end{array}$ \\
\hline $\begin{array}{l}\text { Avoided } \\
\text { oil/gas } \\
\text { heating }\end{array}$ & $\begin{array}{l}\left.\text { Avoided burdens }=\mathrm{Mg} \mathrm{DM} \mathrm{x} \mathrm{m} / \mathrm{Mg} \mathrm{CH}_{4} \text { yield (Table } 3\right) \times 0.67 \mathrm{~kg} / \mathrm{m}^{3}-1 \% \text { digester } \\
\text { loss }-0.5 \% \text { CHP slip x } 50 \mathrm{MJ} / \mathrm{kg} \mathrm{LHV} \times 45 \% \text { CHP heat efficiency }-33 \% \text { parasitic } \\
\text { heat use } \times 30 \% / 27 \% / 0 \% \text { utilisation rate small/medium/large scale AD) x oil/gas heat } \\
\text { burdens per MJ heat from Ecoinvent v3.1. Net credit values in Table } 2 \text {. }\end{array}$ \\
\hline $\begin{array}{l}\text { Avoided } \\
\text { NPK } \\
\text { fertiliser } \\
\text { manufacture }\end{array}$ & $\begin{array}{l}\text { Avoided burdens }=\mathrm{Mg} \text { DM x nutrient contents, } \mathrm{kg} / \mathrm{Mg} \text { (Table } 3 \text { ) }- \text { storage } \mathrm{NH}_{3}-\mathrm{N} \\
\text { loss (above) x MANNER NPK availability factors (Nicholson et al., 2013) x } \\
\text { Ecoinvent v3.1 burdens for ammonium-nitrate, triple superphosphate and potassium } \\
\text { chloride expressed per kg N, P and K. Net credit values in Table } 2 \text {. }\end{array}$ \\
\hline $\begin{array}{l}\text { Avoided } \\
\text { NPK } \\
\text { fertiliser } \\
\text { application }\end{array}$ & $\begin{array}{l}\text { Avoided } \mathrm{kg} \mathrm{NH}_{3}-\mathrm{N}=\text { avoided fertilizer } \mathrm{N} \text { application (above) x } 0.017 \text { (Misselbrook } \\
\text { et al., 2012). } \\
\text { Avoided } \mathrm{kg} \mathrm{N} \text { leached = avoided fertilizer } \mathrm{N} \text { application (above) x } 0.10 \text { (Duffy et al., } \\
2013 \text { ). } \\
\text { Avoided } \mathrm{kg} \mathrm{N} \mathrm{N}_{2} \mathrm{O}-\mathrm{N}=\text { avoided fertilizer } \mathrm{N} \text { application (above) x } 0.01+\mathrm{NH}_{3}-\mathrm{N} \text { x } 0.01 \\
+\mathrm{NO}_{3}-\mathrm{N} \times 0.0075 \text { (IPCC, 2006). } \\
\text { Avoided } \mathrm{kg} \mathrm{P} \text { leached = avoided fertilizer P application (above) x } 0.01 \text { (Withers, } \\
\text { 2013). }\end{array}$ \\
\hline
\end{tabular}

150 Burden data for key inputs and processes are summarized in Table 2. Crop cultivation burdens 151 were based on Styles et al. (2015b) for UK cultivation of maize and grass, and from Ecoinvent 152 v3.1 (Weidema et al., 2013) for fodder beet, and for rye as a proxy for "other cereal silage" 153 (Table 2). The effect of possible land transformation caused by displacement of food production 154 by cultivation of biogas crops, or by additional cultivation of wheat to compensate for animal 155 feed diverted to $\mathrm{AD}$, was evaluated for the purposes of uncertainty analyses. The ILUC factors 156 proposed in Styles et al. (2015b) for land transformation at the global agricultural frontier were 157 applied to an area equivalent to $100 \%$ of the area occupied by the biogas or compensatory wheat158 feed crops. These factors equate to a GWP burden of $25 \mathrm{Mg} \mathrm{yr}^{-1} \mathrm{CO}_{2} \mathrm{e}$ and an EP burden of 9.9 $159 \mathrm{~kg} \mathrm{yr}^{-1} \mathrm{PO}_{4} \mathrm{e}$, respectively, per hectare transformed, based on IPCC (2006) default LUC factors 160 (representing a worst case effect). Methodology and embodied burdens are further elaborated in 161 the following sections, and in SI Section 3. 
162 Table 2. Environmental burden data applied to key inputs and processes, for global warming 163 potential (GWP), eutrophication potential (EP) acidification potential (AP) and fossil resource 164 depletion potential (FRDP)

\begin{tabular}{|c|c|c|c|c|c|}
\hline Input/process & Reference unit & $\begin{array}{c}\mathrm{GWP} \\
\mathrm{kg} \mathrm{CO}_{2} \mathrm{e}\end{array}$ & $\begin{array}{c}\mathrm{EP} \\
\mathrm{kg} \mathrm{PO}_{4} \mathrm{e}\end{array}$ & $\begin{array}{c}\mathrm{AP} \\
\mathrm{kg} \mathrm{SO}_{2} \mathrm{e}\end{array}$ & $\begin{array}{c}\text { FRDP } \\
\text { MJe }\end{array}$ \\
\hline \multicolumn{6}{|l|}{ Crop cultivation burdens } \\
\hline Fodder beet & \multirow{4}{*}{$\mathrm{kg} \mathrm{DM}$} & 0.33 & 0.0022 & 0.0030 & 3.35 \\
\hline Grass silage & & 0.39 & 0.0028 & 0.0025 & 1.93 \\
\hline Maize silage & & 0.19 & 0.0016 & 0.0012 & 0.92 \\
\hline Other cereal silage & & 0.31 & 0.0031 & 0.0029 & 2.83 \\
\hline \multicolumn{6}{|c|}{ (Avoided) upstream burdens for inputs } \\
\hline Ammonium nitrate- $\mathrm{N}$ & $\mathrm{kg} \mathrm{N}$ & 6.10 & 0.0068 & 0.024 & 55.7 \\
\hline Triple superphosphate & $\operatorname{kg~P}$ & 0.889 & 0.020 & 0.016 & 12.5 \\
\hline Potassium chloride $\mathrm{K}_{2} \mathrm{O}$ & $\mathrm{kg} \mathrm{K}$ & 0.42 & 0.0007 & 0.0014 & 6.91 \\
\hline Diesel upstream & $\mathrm{kg}$ & 0.69 & 0.0009 & 0.0062 & 51.6 \\
\hline \multicolumn{6}{|l|}{ (Avoided) field emissions } \\
\hline Fertiliser $\mathrm{N}$ application & $\mathrm{kg} \mathrm{N}$ & 5.12 & 0.054 & 0.035 & - \\
\hline $\begin{array}{l}\text { Manure/digestate } \mathrm{N} \\
\text { application* }\end{array}$ & $\mathrm{kg} \mathrm{N}$ & $\begin{array}{l}5.05- \\
6.92\end{array}$ & $\begin{array}{c}0.034- \\
0.230\end{array}$ & $\begin{array}{c}0.155- \\
0.525\end{array}$ & - \\
\hline $\mathrm{P}$ application & $\mathrm{kg} \mathrm{P}$ & - & 0.031 & - & - \\
\hline Tractor diesel combustion & $\mathrm{kg}$ diesel & 3.06 & 0.0005 & 0.002 & - \\
\hline \multirow{2}{*}{$\begin{array}{l}\text { Tractor-trailer transport } \\
\text { 16-32 tonne Euro IV truck } \\
\text { transport }\end{array}$} & tkm & 0.161 & 0.00025 & 0.0011 & 2.14 \\
\hline & $\mathrm{tkm}$ & 0.134 & 0.00011 & 0.0005 & 1.75 \\
\hline \multicolumn{6}{|l|}{ Biomethane combustion** } \\
\hline CHP combustion & kWhth & & 0.00004 & 0.00045 & \\
\hline EURO V car & vkm & & 0.00003 & 0.0001 & \\
\hline \multicolumn{6}{|l|}{ Replaced fossil energy } \\
\hline $\begin{array}{l}\text { Marginal gas electricity } \\
\text { generation }\end{array}$ & \multirow[t]{2}{*}{$\mathrm{kWh}_{\mathrm{e}}$} & 0.40 & 0.00004 & 0.0010 & 6.55 \\
\hline Coal electricity generation & & 1.02 & 0.0010 & 0.0015 & 16.9 \\
\hline Oil heating & \multirow{2}{*}{$\mathrm{kWh}_{\mathrm{th}}$} & 0.33 & 0.00009 & 0.0006 & 4.50 \\
\hline Gas heating & & 0.25 & 0.00006 & 0.0004 & 3.89 \\
\hline EURO V Diesel car & \multirow{2}{*}{$\mathrm{Vkm}$} & 0.24 & 0.0002 & 0.0007 & 3.33 \\
\hline EURO V Petrol car & & 0.28 & 0.0002 & 0.0008 & 3.83 \\
\hline \multicolumn{6}{|c|}{ Replaced waste management $* * *$} \\
\hline $\begin{array}{l}\text { Animal feed (avoided } \\
\text { wheat cultivation) }\end{array}$ & \multirow{5}{*}{$\begin{array}{c}\mathrm{kg} \text { DM } \\
\text { biowaste }\end{array}$} & -0.58 & -0.0071 & -0.0041 & -3.04 \\
\hline Field residue & & -0.16 & 0.0035 & -0.0003 & -0.51 \\
\hline Landfilling & & 1.99 & 0.0006 & 0.0016 & -6.01 \\
\hline In-vessel composting & & 0.66 & 0.0032 & 0.0070 & 1.92 \\
\hline Incineration & & 0.00 & 0.0009 & 0.0005 & 0.00 \\
\hline
\end{tabular}


*based on MANNER-NPK model (Nicholson et al., 2013); **CHP and upgrade methane slip accounted for separately. $* * *$ these counterfactual waste management options, avoided by AD treatment of various waste fractions, are explained in SI section 3.
165

166

167

168

169

170

171 feedstock material (Table 3), is summarized in SI Section 2. In line with the CLCA objectives,

172 only net additional transport incurred for each feedstock was used to calculate biogas sector

173 transport burdens. Thus, no additional transport burdens were incurred for manure feedstocks

174 compared with baseline manure management, nor for onsite processing of wet wastes. Crops and

175 other waste feedstocks incurred transport burdens of 5 and $50 \mathrm{~km}$, respectively. In all cases

176 except for manures it was assumed that digestates incurred additional transport burdens of $5 \mathrm{~km}$

177 for field application.

179 Table 3. Quantities and characteristics of feedstocks digested in UK biogas plants, in declining 180 order of magnitude, categorized according to five categories: food waste (FW), crop waste (CW),

181 other waste (OW), crops (C) and manures (M)

Feedstock

Digest
ate




\begin{tabular}{|c|c|c|c|c|c|c|c|c|c|c|}
\hline 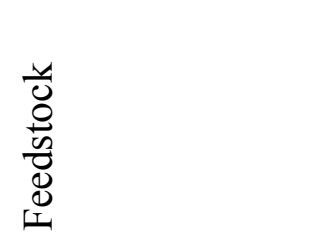 & 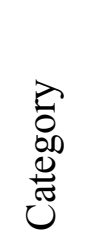 & 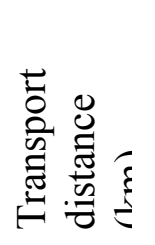 & 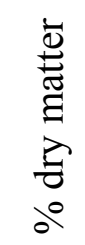 & 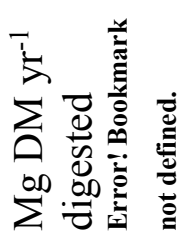 & 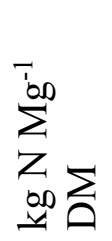 & 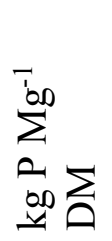 & 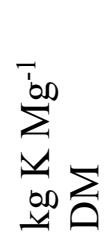 & 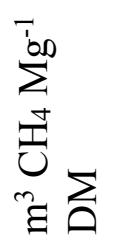 & 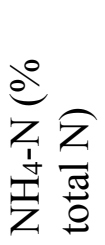 & \\
\hline Food waste & FW & 50 & 26 & 462,410 & 27 & 11.8 & 4.1 & 369 & 80 & $\begin{array}{l}\text { WRAP (2010); Defra } \\
\text { (2014) }\end{array}$ \\
\hline Maize silage & $\mathrm{C}$ & 5 & 30 & 158,130 & 14.1 & 6.2 & 3.8 & 332 & 37 & FNR (2010) \\
\hline $\begin{array}{l}\text { Other industrial } \\
\text { waste }\end{array}$ & OW & 50 & 26 & 44,647 & 16.8 & 7.3 & 5.6 & 323 & 59 & $\begin{array}{l}\text { FNR (2010); WRAP } \\
\text { (2009) }\end{array}$ \\
\hline $\begin{array}{l}\text { Other whole-crop } \\
\text { cereal silage }\end{array}$ & $\mathrm{C}$ & 5 & 33 & 43,486 & 18.6 & 8.1 & 8.2 & 312 & 37 & FNR (2010) \\
\hline Brewery waste & OW & 0 & 22 & 41,949 & 34 & 14.8 & 5.1 & 297 & 59 & Wellinger et al. (2013) \\
\hline Cattle slurry & M & 0 & 10 & 40,296 & 40.7 & 17.8 & 14.9 & 140 & 75 & Defra (2010) \\
\hline Crop waste & CW & 5 & 17 & 37,027 & 26 & 11.4 & 6.6 & 240 & 59 & $\begin{array}{c}\text { Defra (2012); Deublein et } \\
\text { al. (2008) }\end{array}$ \\
\hline Grass silage & $\mathrm{C}$ & 5 & 25 & 32,944 & 21.5 & 9.4 & 6.6 & 306 & 37 & Styles et al. (2015a) \\
\hline $\begin{array}{l}\text { Animal } \\
\text { processing waste }\end{array}$ & OW & 0 & 15 & 26,338 & 20.4 & 8.9 & 19.4 & 199 & 59 & $\begin{array}{c}\text { FNR (2010); Wellinger et } \\
\text { al. (2013) }\end{array}$ \\
\hline Beet & $\mathrm{C}$ & 5 & 23 & 20,206 & 19.4 & 8.5 & 6.9 & 313 & 37 & Styles et al. (2015b) \\
\hline Bakery waste & OW & 50 & 61 & 18,755 & 37 & 16.2 & 1.7 & 304 & 59 & Poeschl et al. (2012) \\
\hline Pig slurry & M & 0 & 4 & 8,809 & 99.3 & 43.4 & 37.3 & 283 & 80 & FNR (2010); Defra (2010) \\
\hline Poultry slurry & M & 0 & 10 & 6,979 & 55.4 & 24.2 & 62.2 & 225 & 75 & FNR (2010); Defra (2010) \\
\hline Waste starch & OW & 0 & 3.3 & 6,200 & 45 & 19.6 & 23.2 & 703 & 59 & $\begin{array}{l}\text { FNR (2010); Fang et al. } \\
\text { (2011) }\end{array}$ \\
\hline Poultry litter & M & 0 & 40 & 5,943 & 50.2 & 21.9 & 34.6 & 225 & 75 & FNR (2010); Defra (2010) \\
\hline $\begin{array}{l}\text { Cheese } \\
\text { processing }\end{array}$ & OW & 0 & 5 & 3,769 & 25 & 10.9 & 6.3 & 309 & 59 & $\begin{array}{l}\text { FNR (2010); Wellinger et } \\
\text { al. (2013) }\end{array}$ \\
\hline
\end{tabular}

182

183 2.4. Plant operating assumptions

184 As of April 2014, 138 non-WWTP AD plants were operational in the UK (NNFCC, 2014a). An

185 anonymous postal questionnaire was sent to the $78 \mathrm{AD}$ plants for which postal addresses could

186 be found online. The questionnaire contained 30 questions over five sections, covering:

187 feedstock characteristics and quantities; heat and electricity generation and use; digestate storage

188 and application details; operational issues such as maintenance requirements; operator 
189 perspectives on $\mathrm{AD}$ based on their experience. Twenty-four responses were received to the 190 questionnaire survey relating to 26 biogas-CHP plants which were categorised according to size 191 as per NNFCC (2014a): eight small plants (50-250 kWe); seven medium-sized plants (350-500 $192 \mathrm{kWe}) ; 11$ large plants $(\geq 1000 \mathrm{kWe})$. Not all respondents answered all questions, leaving a 193 reduced number of data points for some parameters. Key results from the survey are summarised 194 in Table SI1.1 and Figure 2.

195 Electricity conversion efficiency $\left(\eta_{\text {elec }}\right)$ was estimated by dividing reported gross electricity 196 generation ( $\left.\mathrm{G}_{\text {elec }}, \mathrm{MJ}\right)$ by the lower heating value (LHV) of biomethane produced (LHV biomethane, 197 MJ), which was estimated based on feedstock inputs (Qfeedstock, $\mathrm{Mg}$ ) for feedstocks i-n, using 198 estimated default biomethane yields ( $Y_{\text {biomethane, }} \mathrm{m}^{3} \mathrm{Mg}^{-1}$ ) reported in Table 3, a density of 0.67 $199 \mathrm{~kg} \mathrm{~m}^{-3}$ and a LHV of $50 \mathrm{MJ} \mathrm{kg}^{-1}$ for methane:

$200 \quad \prod_{\text {elec }}=G_{\text {elec }} /\left[\Sigma\left(Q_{\text {feedstock i-n }} \times Y_{\text {biomethane i-n }}\right) \times 0.67 \times 50\right]$.

201 Values ranged from 0.27 to 0.57 (Figure 2), representing variation in actual net biomethane 202 yields and CHP transformation efficiencies. 


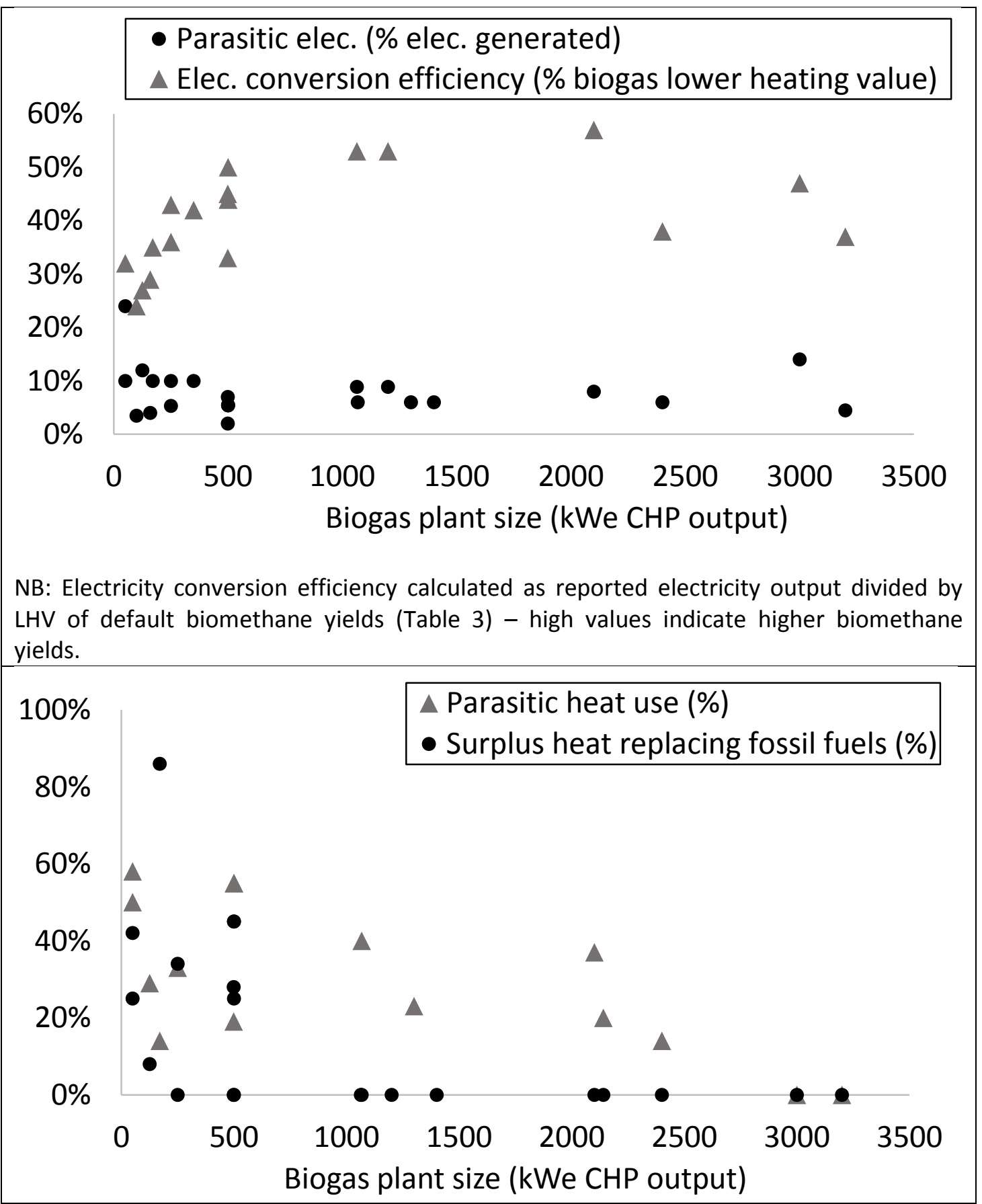

Figure 2. Key operational parameters plotted against AD-CHP electricity generating capacity $(\mathrm{kWe})$

207 Non-parametric Kruskal-Wallis analyses were undertaken in IBM SPSS Statistics 22 to test for 208 differences in the distribution of values across AD plant size categories for the following 209 parameters: estimated biomethane electricity conversion efficiency, parasitic electricity 
210 requirement, parasitic heat requirement, and the fraction of CHP heat output that replaces fossil-

211 fuel heating. Statistically significant size effects were confirmed for electricity conversion

212 efficiency $(\mathrm{p}=0.007)$ and the fraction of CHP heat output replacing fossil fuel heating $(\mathrm{p}=$

213 0.015) (Table SI1.1; Figure 2). Median values for each size category were applied in the national

214 LCA evaluation for these parameters. Estimated biomethane-to-electricity conversion

215 efficiencies (Figure 2; Table SI1.1) indicate that biomethane yields vary from default values

216 presented in Table 3 depending on plant size. Based on median conversion efficiencies (Table

217 SI1.1) and assuming constant CHP electrical efficiencies of $35 \%$ and $40 \%$ for small and

218 medium/large scale biogas plants, respectively (Bacenetti et al., 2013), biomethane yields in

219 Table 3 were multiplied by $0.91,1.10$ and 1.19 in order to calculate biomethane yields for small,

220 medium and large plants, respectively, in the national LCA. Fractions of CHP electricity and

221 heat output required to run the AD plant (parasitic loads), were not statistically different across

222 size categories (Table SI1.1), so overall median values were used for all sizes of biogas-CHP

223 plant considered in the national LCA evaluation.

225 2.5. Counterfactual fate of feedstock

226 Table 4 summarises counterfactual fates of all feedstocks, or in the case of crop feedstocks, the

227 land required to cultivate the crops. Modelling of counterfactual fates was largely based on

228 Styles et al. (2015a;b) and Ecoinvent v3.1 (Weidema et al., 2013), and is elaborated in SI Section

229 3. Table 1 and table 2 summarise methodology and results for key counterfactual (avoided)

230 burdens. Important aspects are mentioned below. 
231 Table 4. Feedstock quantities modelled according to specified permutations of counterfactual

232 management and digestate storage options storage across three biogas-plant-size categories

\begin{tabular}{|c|c|c|c|c|c|c|c|c|c|}
\hline \multirow{2}{*}{ Feedstock } & \multicolumn{3}{|c|}{ Small ( $\leq 250 \mathrm{kWe})$} & \multicolumn{3}{|c|}{ Medium (>250 $\leq 500 \mathrm{kWe}$ ) } & \multicolumn{3}{|c|}{ Large (>500 kWe) } \\
\hline & 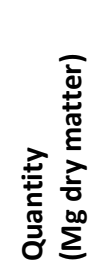 & 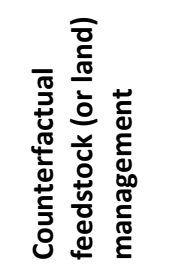 & 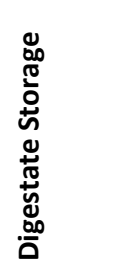 & 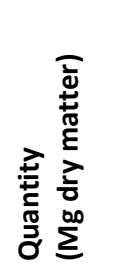 & 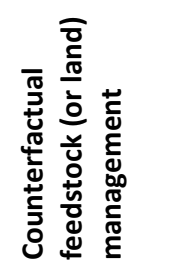 & 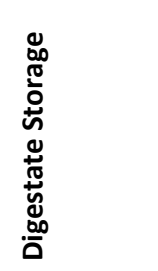 & 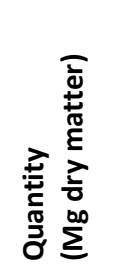 & 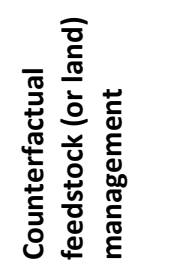 & 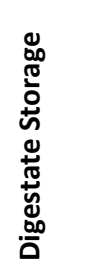 \\
\hline Food waste & & & & $\begin{array}{l}58,664 \\
9,550\end{array}$ & $\begin{array}{l}\text { In-vessel } \\
\text { composting } \\
\text { In-vessel } \\
\text { composting }\end{array}$ & $\begin{array}{l}\text { Lagoon } \\
\text { Open tank }\end{array}$ & $\begin{array}{l}275,937 \\
118,259\end{array}$ & $\begin{array}{l}\text { In-vessel } \\
\text { composting } \\
\text { In-vessel } \\
\text { composting }\end{array}$ & $\begin{array}{l}\text { Lagoon } \\
\text { Sealed } \\
\text { tank }\end{array}$ \\
\hline $\begin{array}{l}\text { Other industrial } \\
\text { waste }\end{array}$ & & & & $\begin{array}{l}5,664 \\
922\end{array}$ & $\begin{array}{l}\text { In-vessel } \\
\text { composting } \\
\text { In-vessel } \\
\text { composting }\end{array}$ & $\begin{array}{l}\text { Lagoon } \\
\text { Open tank }\end{array}$ & $\begin{array}{l}26,642 \\
11,418\end{array}$ & $\begin{array}{l}\text { In-vessel } \\
\text { composting } \\
\text { In-vessel } \\
\text { composting }\end{array}$ & $\begin{array}{l}\text { Lagoon } \\
\text { Sealed } \\
\text { tank }\end{array}$ \\
\hline Bakery waste & & & & $\begin{array}{l}2,379 \\
387\end{array}$ & $\begin{array}{l}\text { Animal feed } \\
\text { Animal feed }\end{array}$ & $\begin{array}{l}\text { Lagoon } \\
\text { Open tank }\end{array}$ & $\begin{array}{l}11,192 \\
4,796\end{array}$ & $\begin{array}{l}\text { Animal feed } \\
\text { Animal feed }\end{array}$ & $\begin{array}{l}\text { Lagoon } \\
\text { Sealed } \\
\text { tank }\end{array}$ \\
\hline Brewery waste & & & & $\begin{array}{l}5,322 \\
866\end{array}$ & $\begin{array}{l}\text { Animal feed } \\
\text { Animal feed }\end{array}$ & $\begin{array}{l}\text { Lagoon } \\
\text { Open tank }\end{array}$ & $\begin{array}{l}25,033 \\
10,728\end{array}$ & $\begin{array}{l}\text { Animal feed } \\
\text { Animal feed }\end{array}$ & $\begin{array}{l}\text { Lagoon } \\
\text { Sealed } \\
\text { tank } \\
\end{array}$ \\
\hline Crop waste & 1,137 & $\begin{array}{l}\text { Field } \\
\text { residues }\end{array}$ & $\begin{array}{l}\text { Gas- } \\
\text { tight }\end{array}$ & $\begin{array}{l}4,611 \\
751\end{array}$ & $\begin{array}{l}\text { Field } \\
\text { residues } \\
\text { Field } \\
\text { residues }\end{array}$ & $\begin{array}{l}\text { Lagoon } \\
\text { Open tank }\end{array}$ & $\begin{array}{l}21,690 \\
9,296\end{array}$ & $\begin{array}{l}\text { Field } \\
\text { residues } \\
\text { Field } \\
\text { residues }\end{array}$ & $\begin{array}{l}\text { Lagoon } \\
\text { Sealed } \\
\text { tank }\end{array}$ \\
\hline Cattle manure & $\begin{array}{l}1,201 \\
2,562 \\
4,243\end{array}$ & $\begin{array}{l}\text { Crusted } \\
\text { tank } \\
\text { Open tank } \\
\text { Lagoon }\end{array}$ & $\begin{array}{l}\text { Open } \\
\text { tank } \\
\text { Open } \\
\text { tank } \\
\text { Open } \\
\text { tank } \\
\end{array}$ & $\begin{array}{l}715 \\
1,524 \\
2,525\end{array}$ & $\begin{array}{l}\text { Crusted } \\
\text { tank } \\
\text { Open tank } \\
\text { Lagoon }\end{array}$ & $\begin{array}{l}\text { Open tank } \\
\text { Lagoon } \\
\text { Lagoon }\end{array}$ & $\begin{array}{l}4,129 \\
8,808 \\
14,589\end{array}$ & $\begin{array}{l}\text { Crusted } \\
\text { tank } \\
\text { Open tank } \\
\text { Lagoon }\end{array}$ & $\begin{array}{l}\text { Lagoon } \\
\text { Lagoon } \\
\text { Lagoon }\end{array}$ \\
\hline Maize silage & 5,016 & $\begin{array}{l}\text { Food/feed } \\
\text { production }\end{array}$ & Lagoon & $\begin{array}{l}19,425 \\
3,162\end{array}$ & $\begin{array}{l}\text { Food/feed } \\
\text { production } \\
\text { Food/feed } \\
\text { production }\end{array}$ & $\begin{array}{l}\text { Lagoon } \\
\text { Open tank }\end{array}$ & $\begin{array}{l}91,369 \\
39,158\end{array}$ & $\begin{array}{l}\text { Food/feed } \\
\text { production } \\
\text { Food/feed } \\
\text { production }\end{array}$ & $\begin{array}{l}\text { Lagoon } \\
\text { Sealed } \\
\text { tank }\end{array}$ \\
\hline $\begin{array}{l}\text { Other cereal } \\
\text { silage }\end{array}$ & 1,379 & $\begin{array}{l}\text { Food/feed } \\
\text { production }\end{array}$ & Lagoon & $\begin{array}{l}5,342 \\
870\end{array}$ & $\begin{array}{l}\text { Food/feed } \\
\text { production } \\
\text { Food/feed } \\
\text { production }\end{array}$ & $\begin{array}{l}\text { Lagoon } \\
\text { Open tank }\end{array}$ & $\begin{array}{l}25,126 \\
10,768\end{array}$ & $\begin{array}{l}\text { Food/feed } \\
\text { production } \\
\text { Food/feed } \\
\text { production }\end{array}$ & $\begin{array}{l}\text { Lagoon } \\
\text { Sealed } \\
\text { tank }\end{array}$ \\
\hline Grass silage & 1,045 & $\begin{array}{l}\text { Food/feed } \\
\text { production }\end{array}$ & Lagoon & $\begin{array}{l}4,047 \\
659\end{array}$ & $\begin{array}{l}\text { Food/feed } \\
\text { production } \\
\text { Food/feed } \\
\text { production }\end{array}$ & $\begin{array}{l}\text { Lagoon } \\
\text { Open tank }\end{array}$ & $\begin{array}{l}19,035 \\
8,158\end{array}$ & $\begin{array}{l}\text { Food/feed } \\
\text { production } \\
\text { Food/feed } \\
\text { production }\end{array}$ & $\begin{array}{l}\text { Lagoon } \\
\text { Sealed } \\
\text { tank }\end{array}$ \\
\hline Poultry litter & 838 & $\begin{array}{l}\text { Manure } \\
\text { heap }\end{array}$ & $\begin{array}{l}\text { Open } \\
\text { tank }\end{array}$ & $\begin{array}{l}429 \\
70\end{array}$ & $\begin{array}{l}\text { Manure } \\
\text { heap } \\
\text { Manure } \\
\text { heap }\end{array}$ & $\begin{array}{l}\text { Lagoon } \\
\text { Open tank }\end{array}$ & $\begin{array}{l}2,018 \\
865\end{array}$ & $\begin{array}{l}\text { Manure } \\
\text { heap } \\
\text { Manure } \\
\text { heap }\end{array}$ & $\begin{array}{l}\text { Lagoon } \\
\text { Sealed } \\
\text { tank }\end{array}$ \\
\hline Poultry slurry & 678 & Pit storage & Open & 347 & Pit storage & Lagoon & 1,632 & Pit storage & Lagoon \\
\hline
\end{tabular}




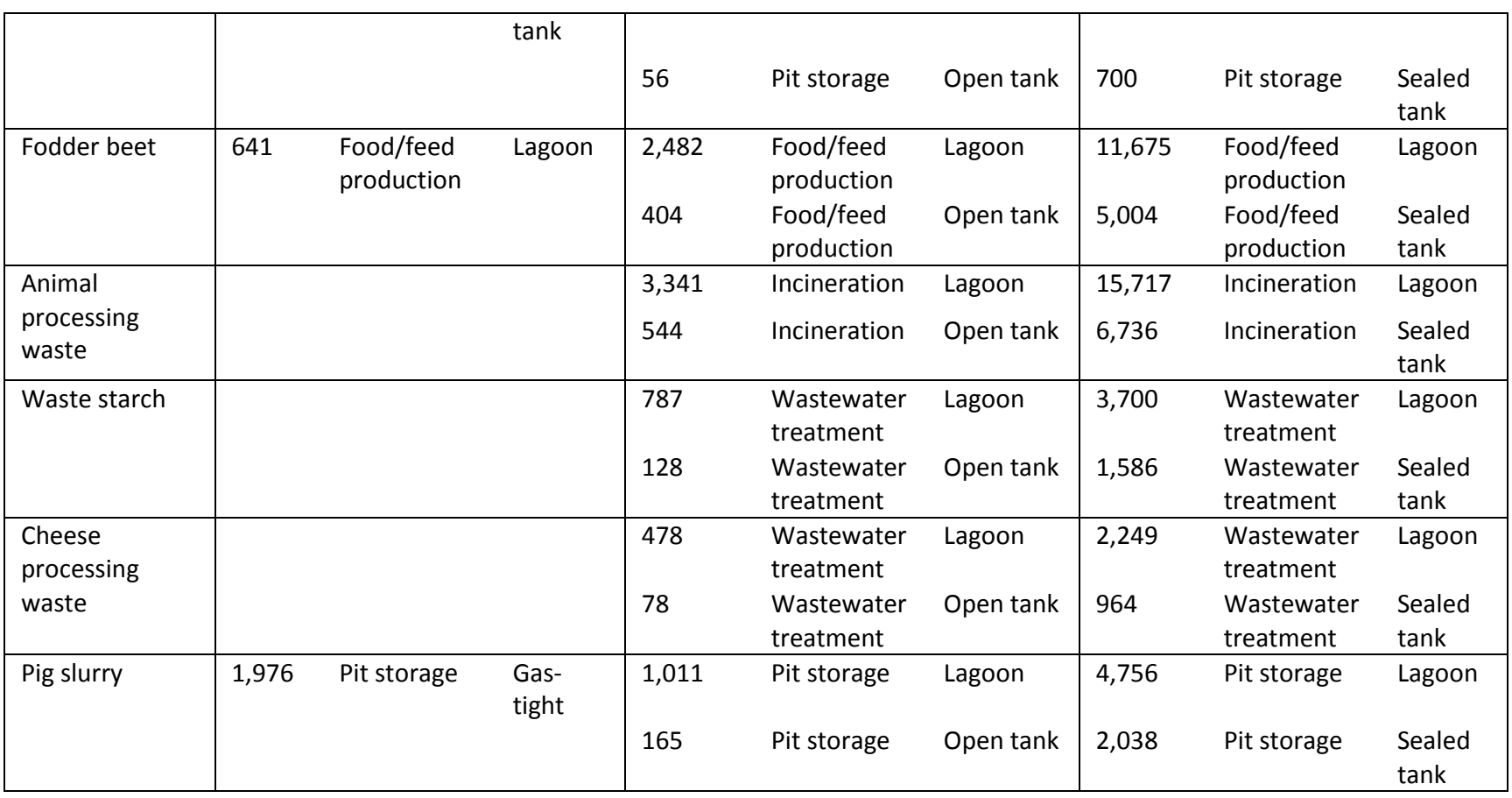

234 Emissions of $\mathrm{CH}_{4}, \mathrm{~N}_{2} \mathrm{O}$ and $\mathrm{NH}_{3}$ during manure storage were based on factors for specific types 235 of storage system given in IPCC (2006) and Misselbrook et al. (2012) (Table 1). Cattle slurry is 236 the dominant manure feedstock (Table 3). Data from the Defra farm practice survey indicate that $23715 \%$ of dairy farms store liquid cattle slurry in a crusted tank, $32 \%$ in an open tank, and $53 \%$ in a 238 lagoon (Defra, 2014a). Counterfactual management of cattle slurry was apportioned across these 239 system types using the above percentages (Table 4). According to Defra (2014a), most "Pigs \& 240 Poultry" farms store their manure in a tank, so it was assumed that all pig and poultry slurry 241 going to $\mathrm{AD}$ was diverted from "pit storage" and "liquid storage", respectively, as defined in 242 IPCC (2006). It was assumed that all solid poultry litter going to AD was diverted from storage

243 in manure heaps. Emissions and NPK fertilizer replacement for counterfactual manure spreading

244 were calculated using the MANNER NPK model (Nicholson et al., 2013), assuming broadcast 245 application in February and April to a spring crop, and June and September to an autumn crop, 246 on a sandy-clay-loam soil. 
247 Medium and large biogas plants digest a wide range of feedstock (Table SI1.1). In-vessel 248 composting was considered to be the marginal type of waste management avoided by AD 249 treatment of separately collected wet organic waste, such as food waste (Table 4). This reflects 250 the dominant fate of separately collected organic waste, and represents a conservative long-term 251 assumption given that a significant fraction of organic waste still goes to landfill in the UK 252 (Mistry et al., 2011). Bakery and brewery wastes are highly valued as animal feeds; 253 conservatively, it was assumed that these waste types were efficiently utilized to substitute 254 wheat-based animal feed on an energy content basis before being diverted to AD (Table 4). 255 Diversion to AD is thus associated with a possible ILUC effect, because additional wheat must 256 be grown to compensate for the lost animal feed (Tonini et al., 2016), as described in SI 3.4. 257 Waste starch and cheese processing wastes are often liquid, with low solids content, and 258 therefore difficult to transport for alternative uses such as animal feed. These wastes were 259 assumed to be disposed of as liquid effluent in the absence of on-site AD, to be treated in an off260 site wastewater treatment plant (Table 4). Conservatively, it was assumed that off-site 261 wastewater treatment involved $\mathrm{AD}$ of sewage sludge, leading to an identical environmental 262 balance to on-site $\mathrm{AD}$ (from a modelling perspective, this leads to no net change in 263 environmental burden whether wastewater is treated in an on-site or off-site AD plant). Owing to 264 health and hygiene regulations surrounding the handling of animal processing waste, incineration 265 was considered the most likely counterfactual fate for this waste stream (Table 4), modelled 266 based on Ecoinvent v3.1 data (Table 2; SI 3.6). Finally, it was assumed that agricultural land 267 used to cultivate biogas-crops would otherwise have been used to produce food or animal feed. 268 Displaced food or feed production may thus incur ILUC. Waste streams going to medium- and 
269 large-scale biogas plants were modelled in quantities determined by the aforementioned statistics

270 and assumptions (Table 4).

271

272 2.6. Digestate management

273 Digestate storage infrastructures for different biogas plant size categories were apportioned as

274 per the distribution across surveyed plants (Table SI1.2). As shown in Table 1, digestate storage

275 infrastructure strongly influences methane and ammonia emissions, although more complete

276 digestion in medium and large-scale plants (indicated by higher electricity yields) constrains

277 methane emissions from digestate storage to $1.5 \%$ of biomethane yield irrespective of storage

278 type in these plants (Lijó et al., 2014b). For medium and large biogas plants, in order to avoid

279 bias associated with apportioning particular feedstocks to particular digestate storage

280 assumptions, each feedstock type was apportioned across the digestate storage practices in

281 proportion to their reported prevalence for each size category (Table 4). Uncertainty around

282 fugitive emissions from digestate storage is high (Adams et al., 2015), represented by an

283 uncertainty range of $\pm 25 \%$ in uncertainty analyses.

284

285 To constrain the number of model runs whilst avoiding bias for particular feedstocks, trailing

286 hose application of digestate was assumed in all cases as a representative, average efficiency

287 technique (Table SI1.1) - approximating to trailing shoe and dribble bar, and between splash

288 plate and injection methods, in terms of $\mathrm{NH}_{3}$ emissions and fertilizer-N-replacement that

289 dominate the environmental profile of digestate application (Table 1). Emissions and NPK

290 fertilizer replacement values were based on results for February and April application to a spring 
291 crop, and June and September application to an autumn crop, on sandy-clay-loam soils, modelled

292 using MANNER NPK (Nicholson et al., 2013) parameterised with relevant digestate nutrient

293 concentrations from Table 3.

295 2.7. Uncertainty and sensitivity analyses

296 Despite the use of detailed survey information to parameterise the LCA modelling, significant

297 uncertainty remains over some biogas-plant factors, such as fugitive emissions, and especially

298 over expanded boundary processes. Digestate storage emissions and fossil energy replacement

299 burdens were varied by $\pm 25 \%$ and $\pm 10 \%$, respectively. High uncertainty over avoided manure

300 management and waste management burdens for individual feedstocks may average out to lower

301 aggregate uncertainty across all feedstock input at the national scale; nonetheless, national

302 aggregate values were varied $\pm 25 \%$. Uncertainty ranges were calculated excluding and including

303 highly uncertain ILUC burdens for all crops and feedstocks diverted from animal feed.

305 Four alternative deployment scenarios were run to test for sensitivity to alternative marginal 306 technologies, and the magnitude of environmental benefit associated with mitigation options.

307 Relevant technologies and associated environmental burdens are summarized in Table 2.

308 Scenario I: The default marginal energy types replaced in the study are natural gas electricity 309 generation via combined cycle turbine and oil heating (DECC, 2012). However, political drivers 310 could lead to coal electricity generation becoming the marginal displaced technology (Finnveden 
311 et al., 2005). The possible effect of this was modelled using burdens for coal electricity

312 generation in the UK taken from Ecoinvent v3.1 (Weidema et al., 2013).

313 Scenario II: Injection of biomethane into the natural gas grid was evaluated as an alternative to

314 CHP in a simple scenario in which $90 \%$ of biogas produced nationally in medium and large scale

315 biogas plants was upgraded and injected into the grid (10\% combusted in onsite CHP to provide

316 heat and power for the biogas plant). Environmental credits were calculated as avoided natural

317 gas heating burdens from Ecoinvent v3.1 whilst incurred post-digestion burdens were calculated 318 assuming 1.4\% $\mathrm{CH}_{4}$ slip during biomethane upgrading (Ravina \& Genon, 2015) and $\mathrm{NO}_{\mathrm{x}}$ 319 emissions from biomethane combustion in boilers taken from Ecoinvent v3.1.

320 Scenario III: The use of upgraded biomethane as a transport fuel was also considered, based on 321 vehicle-km direct burdens only for large Euro $\mathrm{V}$ diesel cars powered by biomethane, taken from 322 Ecoinvent v3.1, and avoiding double-counting of upstream burdens for biomethane production 323 calculated in the $L C A D$ EcoScreen tool. Environmental credits were calculated as vehicle-km 324 burdens for large Euro V cars powered by diesel, taken from Ecoinvent v3.1, and assuming $1 \mathrm{MJ}$ 325 of biomethane replaces $0.75 \mathrm{MJ}$ diesel on a LHV basis (VTT, 2012).

326 Scenario IV: The environmental improvement associated with the retro-fitting of floating covers 327 to all open digestate stores was explored, assuming an $80 \%$ reduction in $\mathrm{NH}_{3}$ emissions (FNR, 328 2010). 
331 Results for the environmental balance of the UK biogas sector in 2014 were conservatively

332 extrapolated to projected deployment in 2017, assuming that all existing plants and $40 \%$ of

333 planned AD plants (NNFCC, 2014b) would be operational in that year. The environmental

334 balance for each feedstock in 2014 was scaled up according to the ratio of tonnage for each

335 feedstock in 2017 versus 2014 (Table SI2.1). This assumes a similar pattern of deployment

336 across plant sizes in 2017 to 2014, and continued dominance of CHP for energy conversion.

\section{RESULTS AND DISCUSSION}

340 In 2014, deployment of $\mathrm{AD}$ in the UK reduced GHG emissions by $653 \mathrm{Gg} \mathrm{CO}_{2} \mathrm{e}$, or $251 \mathrm{Gg}$

$341 \mathrm{CO}_{2} \mathrm{e}$ if worst case ILUC from crop feedstock and diversion of residues from animal feed is 342 accounted for, with a probable range of 238 to $755 \mathrm{Gg} \mathrm{CO}_{2} \mathrm{e}$ after propagating major 343 uncertainties (Table 5). The main GWP credits, in order of decreasing magnitude, were 344 substitution of marginal grid electricity, avoided waste management, substitution of fertilisers 345 and avoided manure management (Figure 3; Table SI4.1). Crops represented 27\% of feedstock 346 DM and contributed $2 \%$ towards GWP savings excluding ILUC, or cancelled out $42 \%$ of GWP 347 savings if worst case ILUC was accounted for. Manures and food waste represented $6 \%$ and $48 \%$ 348 of feedstock DM, and contributed $16 \%$ and $76 \%$ towards GWP savings from $\mathrm{AD}$, respectively

349 (Figure 4). The digestion of brewery waste and bakery waste increased GWP burdens by 11.5

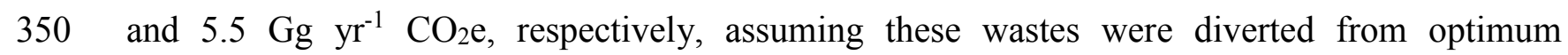


351 counterfactual use as animal feed, thus incurring compensatory cultivation of wheat feed. If this 352 compensatory wheat cultivation in turn incurs ILUC, then net GWP burdens for these two waste

353 fractions increase to 90.2 and $40.6 \mathrm{Gg} \mathrm{yr}^{-1}$, respectively. The conservative assumption that liquid 354 cheese processing waste and waste starch were diverted from treatment in centralized WWTP 355 AD plants meant that these feedstocks had no net effect on the environmental balance of the non356 WWTP AD sector. Aside from crop cultivation and possible ILUC, main GWP burdens arose 357 from digestate storage, digestate application, and fugitive $\mathrm{CH}_{4}$ leakage during fermentation 358 (Figure 3; Table SI4.1).

359 Anaerobic digestion increased national annual eutrophication and acidification burdens by 2.6 $360 \mathrm{Gg} \mathrm{PO}_{4} \mathrm{e}$ and $11.4 \mathrm{Gg} \mathrm{SO}_{2} \mathrm{e}$ in 2014 excluding ILUC, with ranges of 1.8 to $3.4 \mathrm{Gg} \mathrm{PO}_{4} \mathrm{e}$ and 8.1 361 to $14.6 \mathrm{Gg} \mathrm{SO}_{2} \mathrm{e}$ after propagating major uncertainties (Table 5). Crops, food waste and "other 362 waste" contributed approximately one third each towards eutrophication burdens, whilst food 363 waste accounted for $55 \%$ of acidification burdens owing to its volume and high $\mathrm{NH}_{3}$ emissions 364 from $\mathrm{NH}_{4}$-rich digestate (Figure 4). Notably, digestate storage was found to be a particular 365 hotspot in our analyses, owing to the use of $\mathrm{NH}_{4}$-driven $\mathrm{NH}_{3}$ emission factors for different 366 storage systems (Table 1; Misselbrook et al., 2012) which appear to be significantly higher than 367 digestate storage emission factors reported by Amon et al. (2006) and applied in other studies 368 (e.g. Lijó et al., 2014a;b). The higher $\mathrm{NH}_{4}-\mathrm{N}$ content and $\mathrm{pH}$ of digestates compared with slurries 369 would suggest that $\mathrm{NH}_{3}$ emissions from digestate storage are likely to be higher than for slurry 370 storage.

371 Despite their relatively small quantities, brewery and bakery waste made significant net 372 contributions to the eutrophication burden of the AD sector, again owing to diversion from 373 animal feed, incurring additional wheat cultivation. Dominant contributing processes were, in 
374 order of decreasing importance, digestate storage, digestate application, cultivation (for EP only),

375 and CHP combustion (Figure 3; Table SI4.1 and Table SI4.2). Avoided manure management and

376 avoided waste management represented the main acidification and eutrophication credits.

377 In 2014, AD reduced fossil resource depletion in the UK by $9.8 \mathrm{PJe}$, with a range of 8.9 to 10.8

378 PJ (Table 5). Food waste and crops contributed $62 \%$ and $22 \%$, respectively, towards fossil

379 resource savings. Burdens arising from waste transport, crop cultivation and avoided manure

380 application (avoided fertilizer replacement) were small compared with the credits arising from

381 substitution of marginal grid electricity (Figure 3; Table SI4.4). Digestate application (fertilizer

382 replacement) and the avoidance of waste composting generated small but significant FRDP

383 credits (Figure 3).

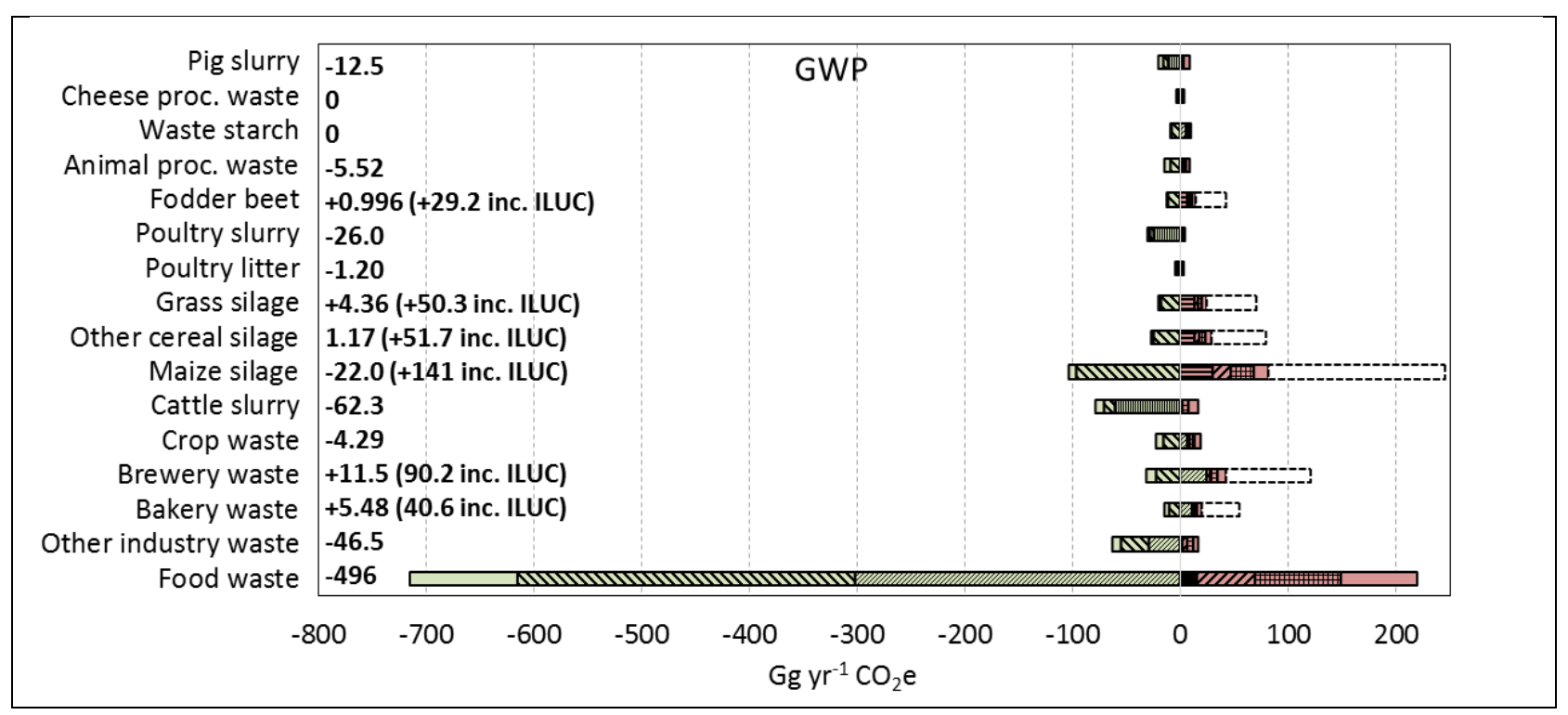




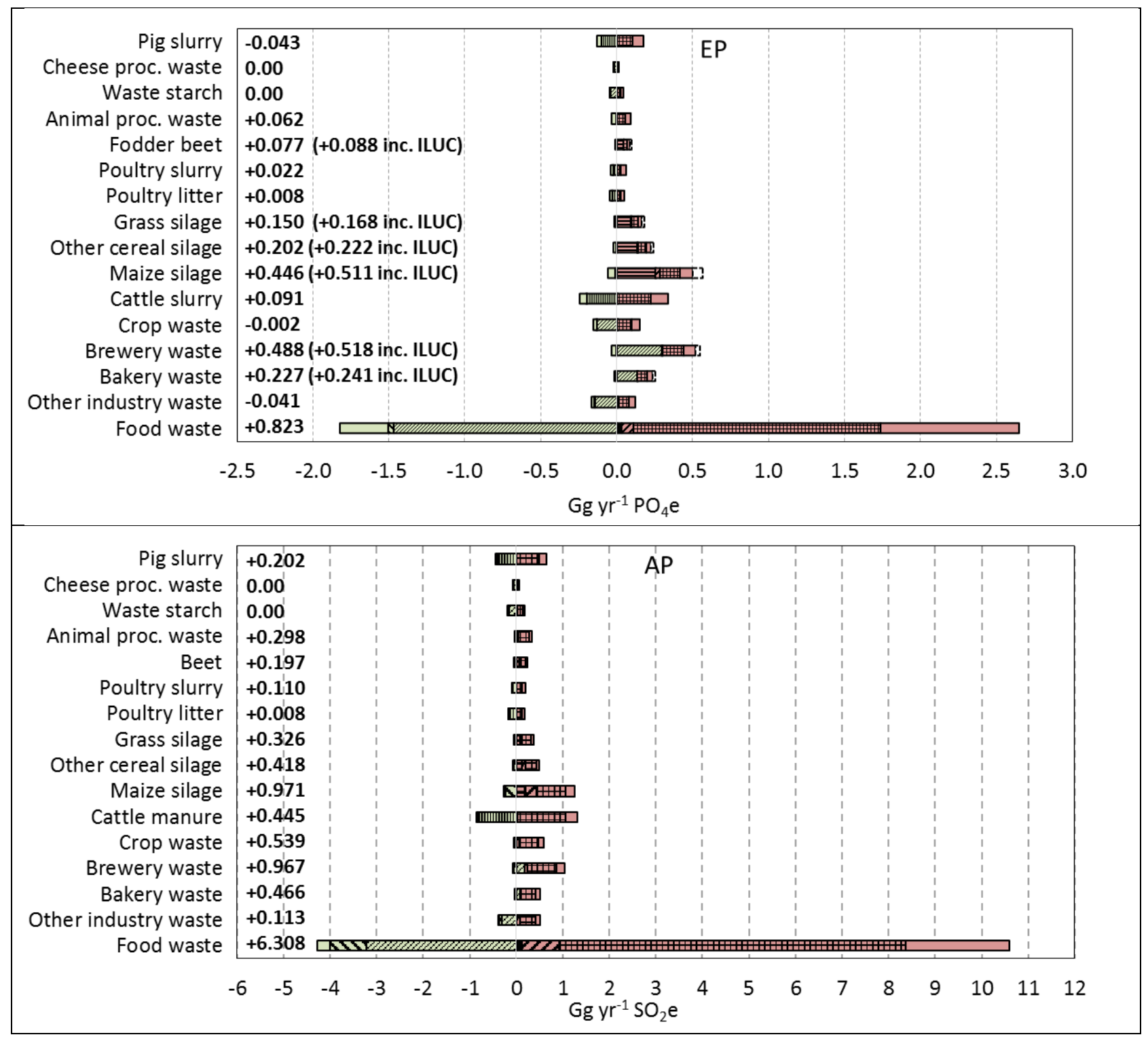




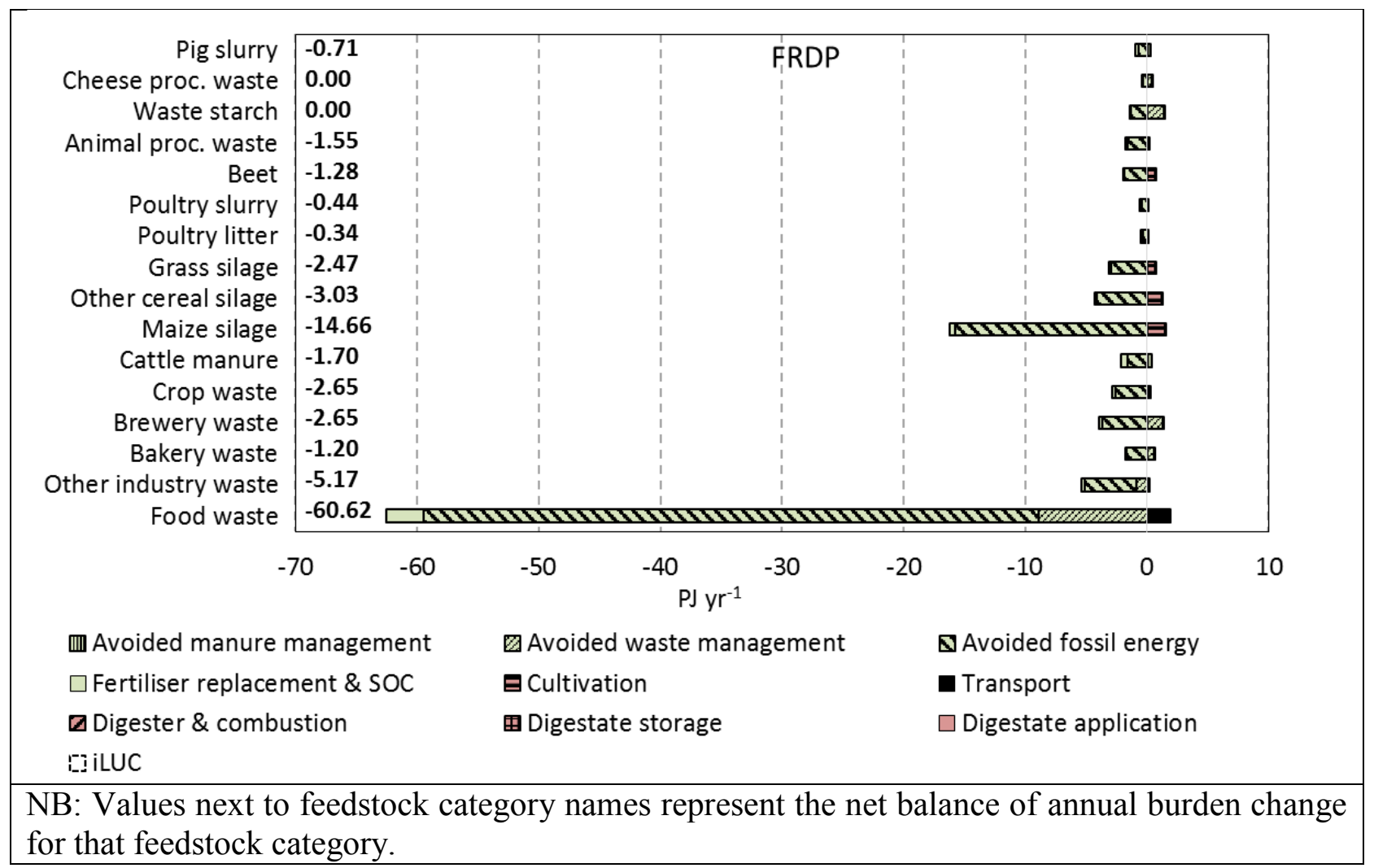

384 Figure 3. Environmental burden changes arising from deployment of anaerobic digestion in the

385 UK in the year 2014, by feedstock type and by (avoided) process (negative values = credits;

386 positive values $=$ burdens; net burdens written next to feedstock name).

3.2. Environmental balance across plant size categories

389 Figure 4 shows the breakdown of sectoral environmental credits and burdens, alongside

390 feedstock input, across the three size categories of biogas plant considered, for the year 2014.

391 Small, medium and large biogas plants accounted for $2 \%, 14 \%$ and $83 \%$, respectively, of

392 feedstock DM input (Table 4 and Table SI2.2). For medium and large biogas plants, the shares of

393 sectoral environmental credits and burdens were similar to the shares of feedstock inputs. For

394 small plants, GWP credits were considerably larger (up to 7\% of national biogas GWP credit, 
395 including ILUC effect) and FRDP credit significantly smaller (1\% of national biogas FRDP

396 credit) than the $2 \%$ feedstock DM input to these plants. AP and EP burden contributions were

397 also significantly smaller than the relative feedstock input. This reflects the environmental

398 profile of the constrained mix of manure (56\% DM), crop waste (5\% DM) and purpose-grown-

399 crop (39\% DM) feedstocks fed in to small biogas plants (Table 4; Table SI1) - leading to large

400 GWP credits for avoided manure storage, but smaller FRDP credits owing to the lower energy

401 conversion efficiency of small scale plants.

402

403

404

405

406

407

408

409

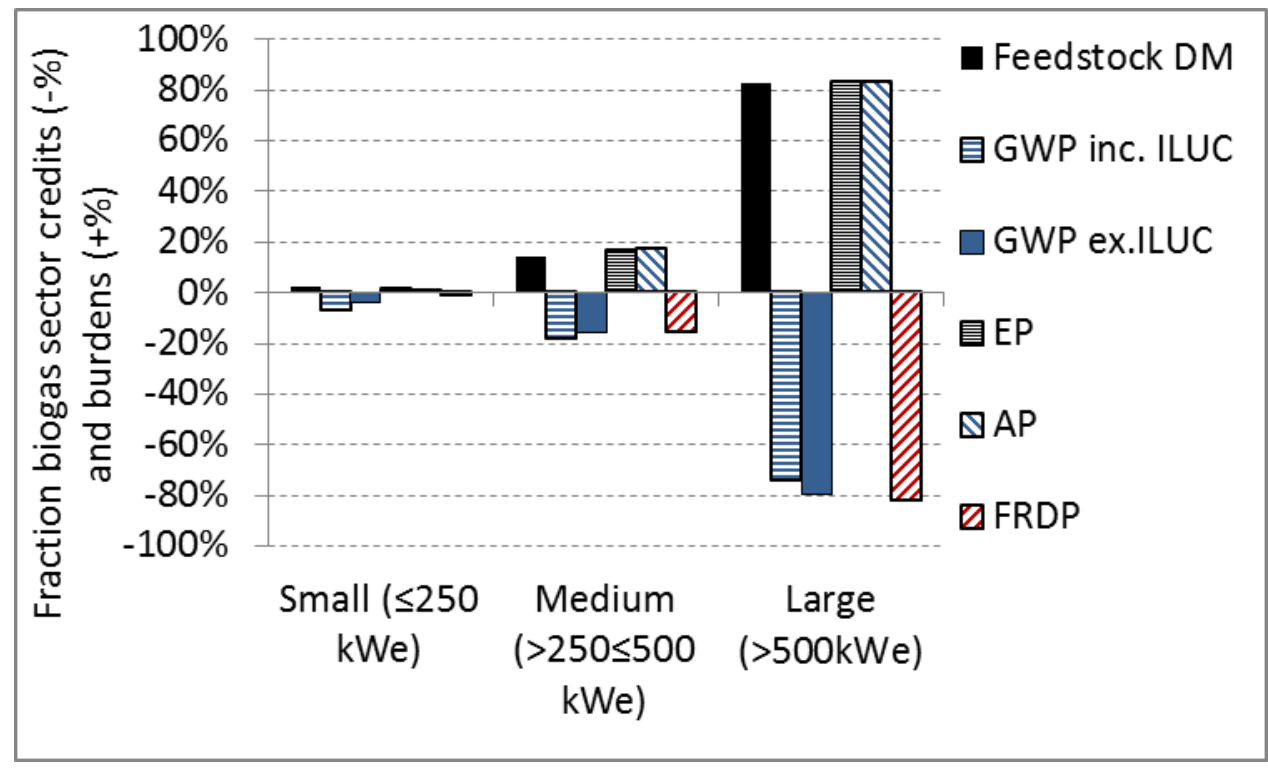

Figure 4. Distribution of sectoral total feedstock input and net environmental credits and burdens across different size categories of AD plant for the year 2014

3.3. Projected national balance of UK biogas sector

In 2014, the AD sector reduced UK total GWP and FRDP burdens by $0.1 \%$ and $0.2 \%$, and increased EP and AP burdens by $0.1 \%$ and $0.5 \%$, respectively (Figure 5). The net GWP reduction is close to zero at the top end of the uncertainty range, primarily owing to highly 
410 uncertain ILUC effects. The quantity of feedstock digested is projected to increase from 5,134

411 Gg fresh matter in 2014 to $12,118 \mathrm{Gg}$ fresh matter in 2017, with a similar composition (Table

412 SI2.1), leading to reductions of $0.3 \%$ and $0.5 \%$ in national GWP and FRDP burdens, and

413 increases of $0.3 \%$ and $1.3 \%$ in national EP and AP burdens, respectively and excluding ILUC

414 effects (Figure 5). Food waste will continue to dominate GWP and FRDP credits and AP burden

415 increases (Figure 5), reflecting its relative volume, avoided composting burdens and $\mathrm{NH}_{3}$

416 emissions from $\mathrm{NH}_{4}$-rich digestate. However, whilst the projected 2017 environmental balance

417 was extrapolated assuming that new biogas plants reflect the current profile of the sector, 34 out

418 of the 415 "in development" biogas plants as of 2014 were biomethane-to-grid plants (NNFCC,

419 2014b). Therefore, according to grid injection performance reported in Table 5, the GHG and

420 FRDP balance of the biogas sector in 2017 may be slightly better than indicated in Figure 5,

421 though within error bar ranges.

422

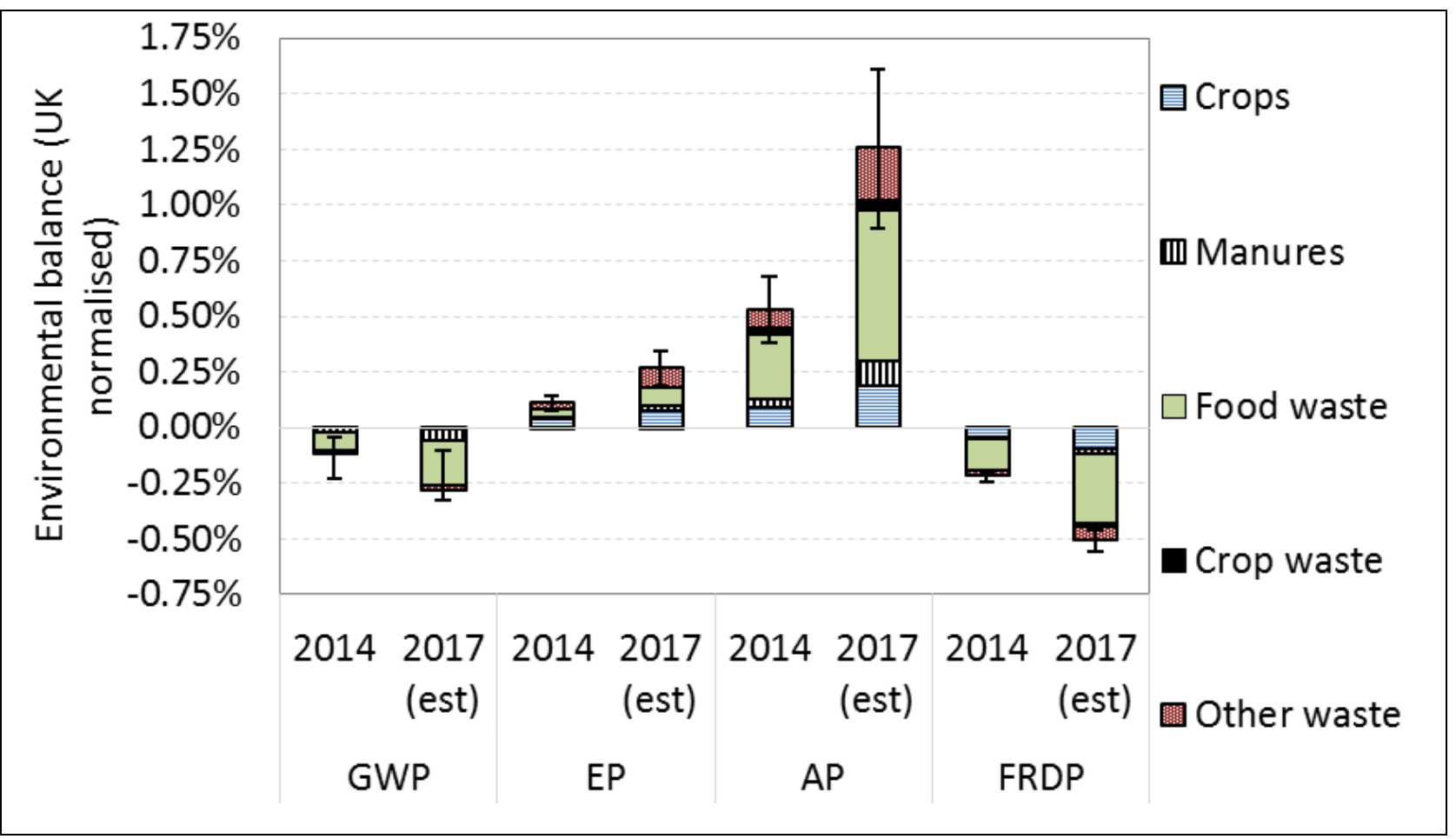


Error bars represent propagated errors for uncertainty ranges shown in Table 5. 2017 values estimated based on NNFCC (2014b) projections.

424 Figure 5. Contribution of feedstock categories to the environmental balance of 2014 and 425 projected (2017) AD deployment in the UK, normalized against UK environmental loadings

\section{3.4. GHG abatement versus renewable energy generation}

427 Projected AD deployment will lead to a net reduction in national annual GHG emissions of $4281,595 \mathrm{Gg}(1.59 \mathrm{Mt}) \mathrm{CO}_{2} \mathrm{e}$, but will still only treat approximately $38 \%$ and $5 \%$, respectively, of

429 national food wastes and manures in 2017. Thus, there remains considerable technical potential

430 to achieve further GHG abatement through targeted expansion of AD. Treating all food waste 431 generated and all handled (stored) manures in the UK through AD could lead to GHG abatement 432 of $11,473 \mathrm{Gg}(11.5 \mathrm{Mt}) \mathrm{CO}_{2} \mathrm{e}$ per year, a 5\% reduction in national GHG emissions, and could 433 avoid $93 \mathrm{PJ}$ of fossil energy consumption. The diversion of other waste streams, such as 434 industrial wastes, towards AD could increase this theoretical potential further. The economic 435 feasibility of digesting a large share of food waste and industrial wastes in large-scale AD plants 436 is high, owing to gate fees, FiT income and economies of scale. However, whilst it may be 437 economically feasible to install biogas plants on large pig, poultry and dairy farms, the 2015 FiT 438 rate of $£ 0.10 \mathrm{kWh}^{-1}$ bioelectricity for biogas plants $\leq 250 \mathrm{kWe}$ capacity is not sufficient to make 439 small-scale manure AD economically viable (Mesa-Dominguez et al., 2015). Using the same 440 CLCA methodology applied here, Mesa-Dominguez et al. (2015) estimated that digestion of all 441 slurry from large UK dairy farms (> 400 milking cows) could lead to GHG abatement of up to $4420.574 \mathrm{Gg} \mathrm{yr}^{-1} \mathrm{CO}_{2} \mathrm{e}$, equivalent to a saving of $3.3 \mathrm{~kg} \mathrm{CO}_{2} \mathrm{e}$ for each $\mathrm{kWh}$ of bioelectricity 443 generated. Even at a high FiT rate of $£ 0.20 \mathrm{kWh}^{-1}$, this would translate into an abatement cost of $444 £ 60$ per $\mathrm{Mg} \mathrm{CO}_{2} \mathrm{e}$. In comparison, large-scale maize-fed $\mathrm{AD}$ leads to net $\mathrm{GHG}$ abatement of just 
$4450.16 \mathrm{~kg} \mathrm{CO}_{2} \mathrm{e} \mathrm{kWh}^{-1}$ (excluding possible ILUC), at a cost of $£ 539 \mathrm{Mg}^{-1} \mathrm{CO}_{2} \mathrm{e}$ at the $2015 \mathrm{FiT}$ rate 446 of $£ 0.087 \mathrm{kWh}^{-1}$ for biogas plants $>500 \mathrm{kWe}$ capacity (OFGEM, 2015). Results presented in

447 Figure 4 confirm that small-scale biogas plants fed with a high share of manure feedstock 448 outperform the overall biogas sector in terms of GHG abatement, despite comparatively low 449 energy yields. Furthermore, although large-scale maize AD-CHP performs comparatively well 450 against other biogas feedstocks in terms of fossil energy substitution, Miscanthus heating pellets, 451 solar PV panels and wind turbines substitute 2, 30 and 90 times more fossil energy per hectare of 452 land utilized, at significantly lower cost (ADAS, 2014; Styles et al., 2015b; Styles, 2015). Thus, 453 there is a case to replace or supplement current FiT and RHI subsidies that incentivize large454 scale crop-fed AD plants with incentives that prioritise efficient GHG abatement through 455 deployment of manure- and waste-fed AD. In the UK, a recent RHI review has proposed that the 456 proportion of biogas originating from crop feedstock be limited to $50 \%$ for individual plants to 457 receive RHI payments on biomethane injected into the grid (DECC, 2016).

459 3.5. Sensitivity analyses and mitigation options

460 The environmental balance of AD deployment in the UK is highly sensitive to the marginal types 461 of waste management and electricity generation replaced. Conservative assumptions regarding 462 the type of waste management option substituted by AD were applied in this study (Table 4), and 463 may underestimate the environmental benefits of AD deployment in the UK. A $25 \%$ change in 464 the aggregate environmental credit attributed to waste management substitution would change 465 EP and GWP results by $13 \%$ and $11 \%$, respectively (Table 5). 
466 If policy intervention could ensure that $\mathrm{AD}$ bioelectricity substitutes electricity generated from 467 coal, rather than natural gas, the environmental balance of national AD deployment would 468 improve substantially; GWP and FRDP credits would more than double (Table 5). Compared 469 with current use of biogas in CHP plants, upgrading biomethane produced in medium- and large470 scale digesters to substitute grid natural gas or transport diesel would increase GHG abatement 471 by $20 \%$ and $38 \%$, respectively. Similar improvements would be achieved for FRDP, with minor 472 reductions in EP and AP burdens. Meanwhile, introducing a regulatory requirement to cover 473 digestate stores could effectively mitigate net eutrophication and acidification burdens arising 474 from $\mathrm{AD}$ (Table 5). 
Table 5. Sensitivity of the annual environmental balance of AD deployment to uncertainty in key parameters, and to alternative deployment scenarios, also expressed as percentage difference from results for 2014 deployment excluding land use change

\begin{tabular}{|c|c|c|c|c|c|}
\hline & & $\begin{array}{c}\mathbf{G W P} \\
\mathrm{Gg} \mathrm{CO}_{2} \mathrm{e} / \mathrm{yr}\end{array}$ & $\begin{array}{c}\text { EP } \\
\mathrm{Gg} \mathrm{PO}_{4} \mathrm{e} / \mathrm{yr}\end{array}$ & $\begin{array}{c}\mathbf{A P} \\
\mathrm{Gg} \mathrm{SO}_{2} \mathrm{e} / \mathrm{yr}\end{array}$ & $\begin{array}{l}\text { FRDP } \\
\text { PJe/yr }\end{array}$ \\
\hline & $\begin{array}{l}\text { Default CHP deployment ex } \\
\text { ILUC }\end{array}$ & -653 & 2.6 & 11.4 & -9.8 \\
\hline \multirow{17}{*}{ 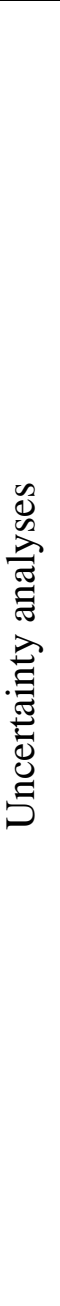 } & $\begin{array}{l}\text { Default CHP deployment } \\
\text { incl. ILUC }\end{array}$ & $\begin{array}{l}-251 \\
-62 \%\end{array}$ & $\begin{array}{l}2.8 \\
6 \%\end{array}$ & $\begin{array}{l}11.4 \\
0 \%\end{array}$ & $\begin{array}{l}-9.8 \\
\mathbf{0 \%}\end{array}$ \\
\hline & $\begin{array}{l}\text { Avoided manure } \\
\text { management }-25 \%\end{array}$ & -629 & 2.7 & 11.7 & -9.9 \\
\hline & & $-4 \%$ & $3 \%$ & $3 \%$ & $\mathbf{0 \%}$ \\
\hline & $\begin{array}{l}\text { Avoided manure } \\
\text { management }+25 \%\end{array}$ & -678 & 2.5 & 11.0 & -9.8 \\
\hline & & $4 \%$ & $-3 \%$ & $-3 \%$ & $\mathbf{0 \%}$ \\
\hline & Avoided waste man $-25 \%$ & $\begin{array}{l}-582 \\
-11 \%\end{array}$ & $\begin{array}{c}2.9 \\
13 \%\end{array}$ & $\begin{array}{l}12.2 \\
8 \%\end{array}$ & $\begin{array}{l}-9.7 \\
-2 \%\end{array}$ \\
\hline & Avoided waste man $+25 \%$ & $\begin{array}{l}-724 \\
\mathbf{1 1 \%}\end{array}$ & $\begin{array}{c}2.3 \\
-13 \%\end{array}$ & $\begin{array}{l}10.5 \\
-8 \%\end{array}$ & $\begin{array}{l}-10.0 \\
\mathbf{2 \%}\end{array}$ \\
\hline & $\begin{array}{l}\text { Digestate storage emissions } \\
-25 \%\end{array}$ & -690 & 1.9 & 8.3 & -9.8 \\
\hline & & $6 \%$ & $-26 \%$ & $-27 \%$ & $0 \%$ \\
\hline & $\begin{array}{l}\text { Digestate storage emissions } \\
+25 \%\end{array}$ & -616 & 3.3 & 14.5 & -9.8 \\
\hline & & $-6 \%$ & $26 \%$ & $27 \%$ & $0 \%$ \\
\hline & Avoided fossil energy $-10 \%$ & -595 & 2.6 & 11.5 & -8.9 \\
\hline & & $-9 \%$ & $0 \%$ & $1 \%$ & $-10 \%$ \\
\hline & Avoided fossil energy $+10 \%$ & -711 & 2.6 & 11.2 & -10.8 \\
\hline & & $9 \%$ & $0 \%$ & $-1 \%$ & $10 \%$ \\
\hline & $\begin{array}{l}\text { Lower bound ex. (inc.) } \\
\text { ILUC }\end{array}$ & $-551(-238)$ & 1.83 & 8.12 & -8.9 \\
\hline & Upper bound & -755 & 3.38 & 14.61 & -10.8 \\
\hline \multirow{5}{*}{ 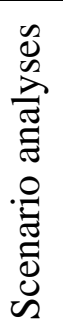 } & I. Replace coal electricity & $\begin{array}{l}-1508 \\
\mathbf{1 3 1 \%}\end{array}$ & $\begin{array}{c}2.3 \\
-11 \%\end{array}$ & $\begin{array}{l}10.6 \\
-6 \%\end{array}$ & $\begin{array}{l}-24.2 \\
146 \%\end{array}$ \\
\hline & $\begin{array}{l}\text { II. Grid injection (M\&L } \\
\text { plants) }\end{array}$ & -786 & 2.6 & 10.6 & -12.2 \\
\hline & & $20 \%$ & $0 \%$ & $-6 \%$ & $24 \%$ \\
\hline & III. Transport (M\&L plants) & -901 & 2.5 & 9.4 & -12.8 \\
\hline & & $38 \%$ & $-6 \%$ & $-17 \%$ & $30 \%$ \\
\hline
\end{tabular}




\begin{tabular}{|ccccc|}
\hline IV. Covered digestate stores & -687 & 0.5 & 1.6 & -9.8 \\
& $\mathbf{5 \%}$ & $\mathbf{- 8 2 \%}$ & $\mathbf{- 8 6 \%}$ & $\mathbf{0 \%}$ \\
\hline
\end{tabular}

\subsection{Summary recommendations}

The UK biogas sector delivers important waste management, renewable energy generation and nutrient recycling services, but requires the cultivation of crops and leads to significant emissions from digestate management. Accounting for these effects across multiple systems at a national level requires an expanded-boundary LCA approach, as concluded by Börjesson and Berglund (2007), Tonini et al. (2012) and Tufvesson et al. (2013). Consequential LCA supplements the detailed attributional LCA studies (Poeschl et al., 2012; Bacenetti et al., 2013; Boulamanti et al., 2013; Whiting and Azapagic, 2014; Lijó et al., 2014b) that provide invaluable evidence to benchmark and optimize the operational efficiency of particular systems. Although involving considerable uncertainty, the application of consequential LCA with conservative (worst case) assumptions and uncertainty ranges provides an evidence base for the role of $\mathrm{AD}$ in national policies on climate change, renewable energy generation and waste management, considering pertinent indirect effects and "unintended consequences".

The UK biogas sector delivers significant savings in GHG emissions and fossil resource depletion through replacement of: (i) grid-electricity; (ii) in-vessel composting of organic wastes; (iii) manure storage. The sector is projected to grow significantly, with FiT and RHI subsidies favouring more energy-efficient large-scale waste- and crop-fed biogas plants. However, there remains a large technical potential for further GHG abatement through expanded digestion of wastes and manures, whilst minimizing the digestion of crops that can lead to carbon leakage via indirect land use change. Our results also show that, where it is possible to use wastes to feed 
livestock (e.g. brewery and bakery wastes), this is a more environmentally efficient option than anaerobic digestion, supporting the conclusions of Tufvesson et al. (2013) and Tonini et al. (2016). Rather than basing FiT and RHI incentives on plant size, these subsidies could be based on feedstock type, with higher subsides for manures, and waste feedstocks that cannot be more efficiently utilized as animal feed, in order to derive maximum environmental benefit from AD. Landfill gate fees are partially responsible for driving expansion of $\mathrm{AD}$; in the longer term, as landfilling of organic wastes is eliminated, differential fees (taxes) for waste management options could be based on their respective environmental efficiencies.

Eutrophication and acidification increases caused by the UK biogas sector are primarily attributable to somewhat uncertain $\mathrm{NH}_{3}$ emissions from digestate management, and fertilizer application to biogas-crops. Covering all digestate stores could largely mitigate burdens caused by $\mathrm{NH}_{3}$ emissions, and would be a simple regulatory control point that could be checked during plant planning and commissioning.

Finally, two thirds of the net heat output from AD-CHP plants is dumped; upgrading biomethane to substitute grid natural gas or transport diesel would considerably improve the overall environmental balance of $\mathrm{AD}$ deployment in the UK. There is some evidence that new RHI subsidies are beginning to encourage the development of biomethane-to-grid plants in the UK (NNFCC, 2014b), but incentives for transport biomethane are so far lacking. Given recent evidence of high NOx emissions from modern diesel engines, there remains a need to evaluate possible air quality and health effects of diesel substitution with biomethane, which could represent an area for cost-effective policy support (simultaneously tackling climate change, energy security and air pollution threats). Biomethane is particularly well suited to powering heavy goods vehicles and buses that are less easily adapted to electric propulsion. 


\section{CONCLUSIONS}

Whilst detailed attributional LCA provides a precise environmental profile of direct AD system effects useful for management decisions, consequential LCA provides a less precise but more complete environmental profile of the $\mathrm{AD}$ sector that captures indirect environmental effects pertinent to policy making and regulation. Through the application of consequential LCA and the use of detailed feedstock input data and plant operating characteristics for the AD sector in the UK, this study highlighted the prevailing effects of counterfactual waste management and indirect land use change over biogas energy conversion efficiency in terms of GHG abatement. Accordingly, climate change policy priorities vis-a-vis AD should be to encourage digestion of food waste and manures, whilst restricting digestion of crop inputs and wastes that could be used as animal feed - even if this involves the deployment of smaller biogas plants with lower energy conversion efficiency for manures. It may be wise to focus on AD policy in terms of waste management, rather than renewable energy generation - there are far more efficient renewable energy options in terms of cost and land requirements. Nonetheless, upgrading the biogas produced for use as a transport fuel would considerably improve the environmental profile of the AD sector, compared with current dominant use for electricity generation and also compared with injection of upgraded biomethane into the gas grid. Biogas plant design, specifically the

prevalence of open tanks and lagoons for digestate storage, is a dominant factor behind significant net eutrophication and acidification loadings from the UK biogas sector. Regulations requiring covered digestate stores, and injection application of digestate, could largely mitigate this problem.

\section{AUTHOR INFORMATION}




\section{Corresponding Author}

*David Styles. Email: d.styles@bangor.ac.uk; Tel.: (+44) (0)1248 382502.

\section{ACKNOWLEDGMENT}

The authors are grateful to the Knowledge Economy Skills Scholarships (KESS) programme, a pan-Wales higher level skills initiative led by Bangor University on behalf of the HE sector in Wales, part funded by the Welsh Government's European Social Fund (ESF) convergence programme for West Wales and the Valleys. The authors would also like to thank AD plant operators who returned questionnaire survey responses and hosted site visits.

Supporting Information. Supporting Information is included in a separate document, under the headings: 1. Survey Results; Methodology; 2. Feedstock Quantities; 3. Counterfactual Fates of Organic Waste Streams; 4. Contribution Analyses.

\section{REFERENCES}

Adams, P.W.R., Mezzullo, W.G., McManus, M.C., 2015. Biomass sustainability criteria: Greenhouse gas accounting issues for biogas and biomethane facilities, Energy Policy, 87, 95-109.

ADAS, Review of Land Use Climate Change: An assessment of the evidence base for climate change action in the agriculture, land and wider food chain sectors in wales. ADAS 2014, Leeds UK.

Amon B., Kryvoruchko V., Amon T. \& Zechmeister-Boltenstern S., 2006. Methane, nitrous oxide and ammonia emissions during storage and after application of dairy cattle slurry 
and influence of slurry treatment. Agriculture, Ecosystems \& Environment, 112, 153 162.

Bacenetti, J., Negri, M., Fiala, M., González-García, S., 2013. Anaerobic digestion of different feedstocks: Impact on energetic and environmental balances of biogas process, Science of The Total Environment, 463-464, 541-551.

Börjesson, P.; Berglund, M., 2007. Environmental systems analysis of biogas systems-Part II: The environmental impact of replacing various reference systems. Biomass and Bioenergy, 31, 326-344.

Boulamanti, A.K.; Maglio, S.D.; Giuntoli, J.; Agostini, A., 2013. Influence of different practices on biogas sustainability. Biomass and Bioenergy, 53, 149-161.

Bywater, A., 2011. A Review of Anaerobic Digestion Plants on UK Farms - Barriers, Benefits and Case Studies. Royal Agricultural Society of England, Warwickshire.

Claus, S.; Taube, F.; Wienfort, B.; Svoboda, N.; Sieling, K.; Kage, H.; Senbayram, M.; Dittert, K.; Gericke, D.; Pacholski, A.; Herrmann, A., 2014. Life-cycle assessment of biogas production under the environmental conditions of northern Germany: greenhouse gas balance. Nitrogen workshop special issue papers, Journal of Agricultural Science, 152, $172-181$.

CML, 2010. Characterisation Factors database available online from Institute of Environmental Sciences (CML). In Universiteit Leiden, Leiden.

DECC, 2012. Valuation of energy use and greenhouse gas (GHG) emissions. Department of Energy and Climate Change, UK Government 2012. Available at: https://www.gov.uk/government/uploads/system/uploads/attachment_data/file/68764/12 2-valuationenergyuseggemissions.pdf Last accessed July 2013.

DECC, 2015. Overview: GHG Inventory summary Factsheet. Department of Energy and Climate Change, London. Available at: https://www.gov.uk/government/uploads/system/uploads/attachment_data/file/442586/O verview_2013_without_Net_traded.pdf 
DEFRA Comparative Life Cycle Assessment of Anaerobic Digestion; 2014. Available from http://sciencesearch.defra.gov.uk/Default.aspx?Menu=Menu\&Module=More\&Location $=$ None $\&$ Completed $=0 \&$ ProjectID $=18631$ (accessed 2 December, 2014).

DEFRA, 2010. Fertiliser Manual RB209. Defra, London.

DEFRA, 2012. Optimising inputs and ouputs from anaerobic digestion processes. Available from http://randd.defra.gov.uk/Default.aspx?Menu=Menu\&Module=More \&Location=None \& Completed=0\&ProjectID=14762 Last accessed 26.03.2016.

DEFRA, 2014a. Greenhouse gas mitigation practices - England Farm Practices Survey. Available at: https://www.gov.uk/government/collections/farm-practices-survey Last accessed 01.06.2015.

DEFRA, 2014b. Comparative Life Cycle Assessment of Anaerobic Digestion. Available from http://sciencesearch.defra.gov.uk/Default.aspx?Menu=Menu\&Module=More\&Location $=$ None $\&$ Completed $=0 \&$ ProjectID=18631 Last accessed 26.03.2016.

Deublein, D., Steinhauser, A., Eds. 2008. Biogas from waste and renewable resourecs: An introduction, WILEY-VCH Verlag GmbH \& Co. KGaA, Weinheim.

EC, 2009. Directive 2009/28/EC of the European Parliament and of the Council of 23 April 2009 on the promotion of the use of energy from renewable sources and amending and subsequently repealing Directives 2001/77/EC and 2003/30/EC. OJEU: L 140/16.

Eurostat, 2016. Population and employment statistics page. Available at: http://appsso.eurostat.ec.europa.eu/nui/show.do Last accessed 15.01.2016

Fang, C.; Boe, K.; Angelidaki, I., Biogas production from potato-juice, a by-product from potato-starch processing, in upflow anaerobic sludge blanket (UASB) and expanded granular sludge bed (EGSB) reactors. Bioresource Technology 2011, 102, 5734-574.

Finnveden, G.; Johansson, J.; Lind, P.; Moberg, A.; 2005. Life cycle assessment of energy from solid waste - part 1: general methodology and results, Journal of Cleaner Production, 13, 213-229.

FNR, 2010. Guide to Biogas: from production to use. Fachagentur Nachwachsende Rohstoffe 


\section{e.V. (FNR), Gülzow-Prüzen.}

Hamelin, L.; Joergensen, U.; Petersen, B.M.; Olesen, J.E.; Wenzel, H., Modelling the carbon and nitrogen balances of direct land use changes from energy crops in Denmark: a consequential life cycle inventory. GCB Bioenergy 2012, 4, 889-907.

Hijazi, O., Munro, S., Zerhusen, B., Effenberger, M., 2016. Review of life cycle assessment for biogas production in Europe, Renewable and Sustainable Energy Reviews, 54, 12911300.

http://www.ipcc-nggip.iges.or.jp/public/2006gl/index.html.

IPCC, 2006. 2006 IPCC Guidelines for National Greenhouse Gas Inventories. IPCC 2006.

Available at:

Lijó, L.; González-García, S.; Bacenetti, J.; Fiala, M.; Feijoo, G.; Lema, J.M.; Teresa Moreira, M.; 2014a. Life Cycle Assessment of electricity production in Italy from anaerobic codigestion of pig slurry and energy crops, Renewable Energy, 68, 625-635.

Lijó, L.; González-García, S.; Bacenetti, J.; Fiala, M.; Feijoo, G.; Moreira, M.T., 2014b. Assuring the sustainable production of biogas from anaerobic mono-digestion, Journal of Cleaner Production, 72, 23-34.

Mesa-Dominguez, E.; Styles, D.; Zennaro, K.; Thompson, P., 2015. Evaluating cost-effective greenhouse gas abatement by small-scale anaerobic digestion, REA \& Bangor University. Available at: http://www.biogas.org.uk/images/upload/news_116_REABangorUnismallscaleADrepor tfinal.pdf Last accessed 26.03.2016.

Misselbrook, T.H., Gilhespy, S.L., Cardenas, L.M., Eds. 2012. Inventory of Ammonia Emissions from UK Agriculture 2011. DEFRA, London.

Mistry, P, Procter, C., Narkeviciute, R., Webb, J., Wilson, L., Metcalfe, P., Solano-Rodriguez, B., Conchie, S., Kiff, B., 2011. Implementation of AD in E\&W Balancing optimal outputs with minimal environmental impacts 2011 (AEAT/ENV/R/3162 April 2011).

Nicholson, F.A.; Bhogal, A.; Chadwick, D.; Gill, E.; Gooday, R.D.; Lord, E.; Misselbrook, T.; Rollett, A.J.; Sagoo, E.; Smith, K.A.; Thorman, R.E.; Williams, J.R.; Chambers, B.J., 
2013. An enhanced software tool to support better use of manure nutrients: MANNERNPK. Soil Use and Management, 29, 473-484.

NNFCC, 2014a. Anaerobic digestion deployment in the United Kingdom. National Non Food Crop Centre, York.

NNFCC, 2014b. Anaerobic digestion deployment in the United Kingdom (updated). National Non Food Crop Centre, York.

OFGEM, 2014. Non-Domestic Renewable Heat Incentive (RHI). Available from: https://www.ofgem.gov.uk/environmental-programmes/non-domestic-renewable-heatincentive-rhi

OFGEM, 2016. Feed-in Tariff Generation \& Export Payment Rate Table for Non-Photovoltaic Installations - FIT Year 6 (1 April 2015 to 31 March 2016); 2015. Available from https://www.ofgem.gov.uk/sites/default/files/docs/2015/07/fit_nonpv_tariff_table_1_october_2015_0.pdf Last accessed 14.03.2016.

Poeschl, M., Ward, S., Owende, P., 2012. Environmental impacts of biogas deployment - Part I: Life Cycle Inventory for evaluation of production process emissions to air. Journal of Cleaner Production, 24, 168-183.

Ravina, M.; Genon, G., 2015. Global and local emissions of a biogas plant considering the production of biomethane as an alternative end-use solution. Journal of Cleaner Production, 102, 115-126.

Rehl, T.; Lansche, J.; Müller, J., 2012. Life cycle assessment of energy generation from biogas-Attributional vs. consequential approach. Renewable and Sustainable Energy Reviews, 16, 3766-3775.

Sleeswijk, W.; van Oers, A.L.; Guinée, J.; Struijs, J.; Huijbregts, M.; 2008. Normalisation in product Life Cycle assessment: An LCA of the Global and European Economic Systems in the year 2000. Science of The Total Environment, 390, 227-240.

Styles, D., 2015. Is bioenergy a sensible use of public money? Presentation delivered at “Talking Food vs Fuel”, Hutchinsons Agronomist Conference 2015, Birmingham, February 2015. 
Styles, D.; Gibbons, J.; Williams, A.P.; Dauber, J.; Urban, B.; Stichnothe, H.; Chadwick, D.; Jones, D.L., 2015b. Consequential life cycle assessment of biogas, biofuel and biomass energy options in an arable crop rotation. Global Change Biology Bioenergy, 7, 13051320

Styles, D.; Gibbons, J.; Williams, A.P.; Stichnothe, H.; Chadwick, D.R.; Healey, J.R., 2015a. Cattle feed or bioenergy? Consequential life cycle assessment of biogas feedstock options on dairy farms. GCB Bioenergy, 7, 1034-1049.

Tonini, D.; Hamelin, L.; Wenzel, H.; Astrup, T., 2012. Bioenergy production from perennial energy crops: a consequential LCA of 12 bioenergy scenarios including land use changes. Environmental Science \& Technology, 46, 13521-13530.

Tonini, D., Hamelin, L., and Astrup, T.F., 2016. Environmental implications of the use of agroindustrial residues for biorefineries: application of a deterministic model for indirect land-use changes. GCB bioenergy, doi: 10.1111/gcbb.12290

Tufvesson, L.M.; Lantz, M.; Börjesson, P., 2013. Environmental performance of biogas produced from industrial residues including competition with animal feed - lifecycle calculations according to different methodologies and standards. Journal of Cleaner Production, 53, 214-223.

VVT (2012). Diesel and compressed natural gas combustion emissions from buses. Available at: http://lipasto.vtt.fi/yksikkopaastot/henkiloliikennee/tieliikennee/henkilo_tiee.htm Last accessed 28.09.2015).

Webb, J., Misselbrook, T.H., 2004. A mass-flow model of ammonia emissions from UK livestock production. Atmospheric Environment 38, 2163-2176.

Weidema, B.. 2001. Avoiding co-product allocation in life-cycle. Journal of Industrial Ecology, $4,11-33$.

Weidema, B.P.; Bauer, Ch.; Hischier, R.; Mutel, Ch.; Nemecek, T.; Reinhard, J.; Vadenbo, C.O.; Wernet, G, 2013. The ecoinvent database: Overview and methodology, Data quality guideline for the ecoinvent database version 3, www.ecoinvent.org 
Wellinger, A., Murphy, J., Baxter, D., 2013. The biogas handbook. IEA, Paris. ISBN-13: 9780 857094988

Whiting, A., Azapagic, A., 2014. Life cycle environmental impacts of generating electricity and heat from biogas produced by anaerobic digestion, Energy, 70, 181-193.

WRAP, 2009. Anaerobic Digestate: Partial Financial Impact Assessment of the introduction of a Quality Protocol for the production and use of anaerobic digestate. WRAP, Oxford.

WRAP, 2010. Food Waste Chemical Analysis. Chemical characterisation of food wastes collected from Welsh Local Authorities for supporting decisions related to anaerobic digestion process design and operation. WRAP, Oxford. 